\title{
Les réformes actuelles de la formation à l'enseignement en France et aux États-Unis: Eléments de mise en perspective socio-historique à partir du concept d'éducation
}

\section{Yves Lenoir}

L'article a pour objet de faire ressortir, en s'appuyant sur une perspective sociohistorique, l'existence de dominances dans la conception des modalités d'actualisation des systèmes de formation à l'enseignement en France et aux États-Unis. Ces dominances découlent de deux traditions de mise en ouvre du système d'éducation. Si ces deux traditions ont une visée commune, l'émancipation des êtres humains, visée directement liée à la fonction du système scolaire dans un État-nation moderne, elles résultent toutefois de dynamiques historico-sociales dis-tinc-tes et elles se sont actualisées différemment dans leur société respective. Dans le contexte des échanges internationaux actuels, caractérisés entre autres par une idéologie néolibérale hégémonique portée par divers organismes et supportée par la puissance américaine, l'introduction d'éléments provenant de la tradition étasunienne dans le système éducatif français vient le perturber en suscitant des ruptures dans sa cohérence interne.

\section{Introduction}

Partout en Occident, du Chili en Suisse romande, en passant par le Brésil, les États-Unis, le Canada ou la Belgique néerlandophone et francophone, s'opèrent à l'heure actuelle, en lien direct avec des réformes des curriculums de l'enseignement primaire et secondaire, de profondes transformations de la formation à l'enseignement. Et toutes ces réformes s'appuient sur une structure conceptuelle identique, du moins en apparence, qui fait appel à la professionnalisation, à l'approche par compétences, à l'approche programme, à la transversalité, à l'interdisciplinarité, sinon à la transdisciplinarité, à l'éducation à la citoyenneté, à la perspective constructiviste, etc. À y regarder de plus près toutefois, une analyse fine devrait permettre de faire ressortir des différences d'interprétations, parfois majeures, que cache un usage largement généralisé des mêmes notions.

Ainsi, on peut noter à titre illustratif l'existence d'un recours en France et aux États-Unis à une terminologie différente pour traiter de certaines questions relatives à l'éducation et à l'enseignement. Par exemple, la documentation scienti- 
fique anglophone en éducation témoigne d'une préoccupation pour la notion de curriculum, alors que la documentation francophone se concentre surtout sur la notion de didactique. Les deux termes existent en parallèle et un no man's land semble les séparer, même si des écrits les utilisent de part et d'autre de l'Atlantique. ${ }^{1}$ Que cache cette différence d'utilisation sémantique? Serait-ce le résultat d'une simple question de traduction? Il semble bien que non, car les deux mots curriculum et didactique existent dans les deux langues et, grosso modo, leurs définitions respectives sont équivalentes dans les dictionnaires non spécialisés. Cependant, la recherche anglophone en éducation n'utilise pas le terme didactique, si ce n'est dans un sens hautement péjoratif ${ }^{2}$, et la francophonie utilisait rarement jusqu'à ces dernières années le terme curriculum, préférant l'appellation plus restreinte de programme d'études parce qu'elle correspondait mieux à la conception d'un cursus scolaire (Audigier, 2001). Autre exemple, Bourdoncle (1993) a fait état de conceptions francophone et britannique distinctes au regard du concept de profession. Et Schnapper (1999) souligne des conceptions fort différentes de la citoyenneté dans différents pays. Alors que l'on fait appel en Europe francophone au concept de représentation sociale, les chercheurs nord-américains anglophones recourent à celui de croyance (belief). Pourquoi en est-il ainsi? Serait-ce une question de traditions de recherche utilisant différents termes pour désigner les mêmes phénomènes? Ou serait-ce que ces usages témoigneraient d'une différence plus fondamentale et substantielle? Plusieurs autres observateurs ont noté cette différence au regard de la conception de l'éducation scolaire sans s'être cependant attardés à l'expliquer en profondeur (Durand, 1994; Forquin, 1989; Trottier, 1987; Van Haecht, 1990).

Alors que des travaux ont porté sur l'étude comparative des systèmes et des curriculums de l'enseignement primaire et secondaire (Ball \& Van Zanten, 1998; Bulle, 1999; Coulby, 2000; Fialaire, 1996; Green, 1990; Gundem \& Hopmann, 1998; Hameyer, Frey, Haft \& Kuebart, 1986; Vaniscotte, 1996; Whitty \& Edwards, 1998) comme de l'enseignement universitaire (Drèze \& Debelle, 1968; Goldring, 2000; Lenoir, 2000a; Lessard \& Tardif, à paraître; Readings, 1996), peu d'études, à notre connaissance - hormis le rapport de Lessard, Lenoir, Martin, Tardif et Voyer (1999) et l'ouvrage de Tardif, Lessard et Gauthier (1998) -, se sont aventurées à ce jour dans cette voie en ce qui regarde la formation à l'enseignement.

Dans une recherche à la fois comparative et prospective parue en 1999, Lessard, Lenoir, Martin, Tardif et Voyer font état de quelques éléments de comparaison des politiques de formation à l'enseignement aux États-Unis, au Canada anglais, en Grande-Bretagne et en France. À l'heure d'une mondialisation des communications, de la culture et des échanges au sein de laquelle s'exercent des processus d'emprunts, d'adaptations et d'acclimatations locales, de métissage des finalités, des politiques, des dispositifs et des pratiques de formation, ces auteurs relèvent qu'il n'est plus possible de concevoir le monde, ainsi que les comparatistes le faisaient traditionnellement, comme un ensemble de sociétés nationales 
ou régionales s'inscrivant dans des environnements fortement différenciés, "chacune apparaissant aux autres comme une entité significativement autonome et historiquement distincte» (Lessard et al., 1999, pp. 5-6). Les interdépendances que tissent les processus d'internationalisation montrent bien cette pénétration dans chacune de ces sociétés, à des rythmes certes différents (Tardif, Lessard \& Gauthier, 1998), de conceptions et de tendances «universalisantes» dont, en éducation, l'universitarisation de la formation à l'enseignement et la professionnalisation du métier d'enseignant constituent deux exemples majeurs.

Autant l'école est en mutation en France (Charlot, 1987; Dubet \& Martuccelli, 1996) et ailleurs dans le monde industrialisé, autant le système de formation à l'enseignement se modifie pour chercher à s'adapter aux nouvelles attentes et exigences sociales, fortement connotées par le vent néolibéral qui souffle sur ces sociétés et par la vision économiste que cette idéologie distille. Ainsi, au modèle d'une éducation fondée sur le développement culturel, en vigueur au $19 \mathrm{e}$ siècle et dans la première moitié du vingtième, s'est substitué un modèle d'école devant viser la préparation à l'emploi. Les formations professionnalisantes, de plus en plus présentes, s'inscrivent dans ce dernier modèle. Toutefois, comme le soulignent Tardif, Lessard et Gauthier (1998), cette convergence internationale repose sur le constat suivant: «la vaste majorité des réformes de la formation des enseignants et les tentatives récentes pour professionnaliser l'enseignement proviennent d'abord des États-Unis. Elles sont reprises ensuite par la plupart des autres pays, avec des décalages chronologiques plus ou moins importants et des adaptations à des contextes sociaux particuliers à chacune des sociétés» (p. 10). En effet, comme le remarque Chauvel (2000), "les systèmes scolaires gardent souvent la marque de leurs origines» (p. 343), ce qui n'empêche leur évolution transformatrice, précise-t-il.

Tardif, Lessard et Gauthier (1998) en arrivent à conclure que «la formation des maîtres vit un déplacement de son centre traditionnel de gravité, passant d'une formation disciplinaire et théorique à une formation davantage pédagogique, réflexive et pratique» (p. 287). Altet $(1994,1998)$ et Lessard, Lenoir, Martin, Tardif et Voyer (1999) parviennent à la même conclusion. Une telle tendance est également mise en exergue par l'Organisation de coopération et de développement économiques (OCDE) dont une de ses enquêtes (1992, 1995, 1997) menée au sein de 12 pays membres révèle que la fonction primordiale de l'institution scolaire est en train de glisser d'une centration sur l'acquisition de savoirs à une préoccupation dirigée vers des questions plus spécifiquement d'éducation et de socialisation. Les objectifs sociaux deviennent de plus en plus une priorité de formation, tant aux yeux des pouvoirs publics, de l'entreprise qu'à celui du grand public.

Quant à Bulle (2000) qui se penche sur les modèles éducatifs britannique, américain et français, elle souligne l'importance de deux principes en vigueur à la fin des années cinquante dans les systèmes éducatifs britannique et américain - le parrainage et la compétition - qui donneraient «les clés de la signification 
des changements de l'école dans les sociétés démocratiques modernes» (p. 366). Elle associe le principe de la compétition à une «absence de valeur intrinsèque conférée aux savoirs enseignés» (p. 367) dans l'enseignement secondaire public des États-Unis. Ainsi plusieurs travaux ont attiré l'attention sur une importance moindre accordée socialement à la transmission des savoirs sur le plan scolaire.

Ce changement profond d'orientation, qui pourrait peut-être être qualifié de paradigmatique, n'est pas sans créer maints dérangements au sein même de divers systèmes d'enseignement, dont le système d'enseignement français. À la fin de son livre sur la professionnalisation des enseignants en France, Lang (1999) met en évidence le malaise actuel que vivraient tout particulièrement les enseignants du secondaire (du second degré) face à une rupture identitaire qui se dessinerait au regard des disciplines scientifiques qui leur ont traditionnellement servi de cadre de référence. Ainsi que le relève Lang, la professionnalisation du métier d'enseignant "ne se réduit plus aujourd'hui à un niveau de culture et de maîtrise des savoirs» (p. 239); elle exige aussi, sinon d'abord, des compétences qui se situent sur un autre plan, en référence avec la pratique. Ailleurs, Lang (2000) parle de rupture de la formation des enseignants face à leur identité professionnelle.

Plusieurs facteurs de divers ordres, de poids et d'ampleur différents, ont été avancés pour expliquer ce changement: demande croissante et diversifiée pour un relèvement du niveau de l'éducation (Organisation for economic co-operation and development/Unesco, 2001), évolution de la demande sociale liée à des transformations structurelles, "massification» et démocratisation de l'école, évolution des théories psychologiques, incapacité des disciplines à répondre aux attentes sociales, émiettement des disciplines, émancipation des formations professionnelles, mondialisation, «marchandisation» de l'école, centration sur des problèmes sociaux (violence, égalité des chances, etc.), nouveaux référents épistémologiques, éclatement du consensus social au regard des finalités éducatives, idéologie néo-libérale vectrice de nouvelles valeurs et de nouveaux modèles de gestion, individualisation exacerbée, etc. En fait, sans nier l'existence d'un débat aux États-Unis au regard de la place des savoirs dans la formation d'un futur citoyen $^{3}$, il faut reconnaître que la question agite tout particulièrement le monde francophone.

Le présent article ${ }^{4}$ a pour objet, non de traiter de ces transformations, sources de déséquilibres et autres émois, désarrois et vicissitudes qui agitent les systèmes d'enseignement occidentaux et qui sont amplement scrutés, mais plutôt de remonter au $18^{\mathrm{e}}$ et $19^{\mathrm{e}}$ siècles afin de rappeler les options sociales qui ont prévalu dans la détermination des finalités socio-éducatives de l'éducation scolaire aux États-Unis et en France et de faire ressortir les dominances qui en ont résulté sur le plan de leur mise en œuvre. L'hypothèse avancée est que ces options ont marqué historiquement l'actualisation des traditions éducatives en France et aux États-Unis et ont sans doute influencé d'autres systèmes d'enseignement anglosaxons et francophones. 
Il existerait historiquement, de notre point de vue, deux perspectives différentes, deux univers distincts s'appuyant sur deux traditions différentes, l'une d'elles, étasunienne, prenant actuellement une expansion portée par des phénomènes liés à la mondialisation de l'économie, certes, mais aussi de la culture (Luke \& Luke, 2000; Warnier, 1999). Ce texte présente l'analyse, menée de façon préliminaire, de ces deux traditions au regard du concept d'éducation, en identifie quelques attributs principaux et leurs modalités d'actualisation dans les systèmes scolaires étasunien et français, et en dégage des impacts et enjeux pour la formation à l'enseignement.

Ces traditions pourraient être caractérisées, l'une, française, de logique du sens, centrée sur le savoir, et l'autre, étasunienne, de logique fonctionnelle, centrée sur le sujet apprenant. Elles se sont constituées, développées et actualisées sur la base de rationnels différents (idéologiques, politiques, économiques, culturels) qui s'inscrivent dans le cadre du développement sociohistorique de chacun des États-nations que sont la France et les États-Unis. Le choix de ces deux sociétés n'est pas seulement lié au fait que la société québécoise - à laquelle l'auteur de cet article participe -, tant sur les plans de la formation que de la recherche, se trouve à la confluence de ces deux traditions; il découle également du fait que, d'un côté, les États-Unis tendent aujourd'hui à exporter leurs modèles culturels tout autant qu'économiques et politiques, que la pression internationale pour la participation à des modèles de formation standardisés est forte et que, de l'autre, le système culturel et éducatif français était profondément ancré jusqu'à une période récente dans un système républicain laïc s'appuyant sur les principes de 1789 (Prost, 1997), guère compatible avec les conceptions nordaméricaines.

Cette différence a, pensons-nous, des implications sur le sens attribué par chaque tradition à différents concepts centraux, sur la manière de concevoir les phénomènes d'enseignement et d'apprentissage, sur la formation à l'enseignement et, plus profondément sur la conception de l'éducation et sur ses modalités d'actualisation. Une telle réflexion a d'autant plus de pertinence que le poids de la mondialisation conduit à une interaction grandissante entre ces deux traditions et leurs composantes (Gundem \& Hopmann, 1998), ce qui n'est pas sans avoir un impact sur elles.

Avant toute présentation, il importe d'insister sur le fait que ce dont il est ici question, pour cerner ces deux traditions, ce n'est pas la description de ce qui se passe ou de ce qui s'est effectivement passé dans la quotidienneté concrète de l'enseignement et dans la mise en ouvre effective des politiques éducatives. Nous traitons, pourrait-on dire, de fictions, mais de fictions bien réelles et opérationnelles, au moins indirectement, car elles ont influencé les fins socio-éducatives et les modalités d'actualisation de ces systèmes. Elles ont servi de toile de fond à un argumentaire qui est resté souvent implicite et elles survivent dans l'imaginaire social. Il s'agit donc bien plutôt d'idéologies, comme le précise Schnapper (1994), en ce sens qu'elles reposent sur des construits sociaux, qu'elles ont été 
élaborées dans un contexte spatiotemporel donné, en opposition à d'autres structures et à d'autres systèmes idéologiques de légitimation. Et, ainsi que le souligne Ansart (1974), le propre d'une idéologie est justement de se présenter comme l'antithèse d'une idéologie, comme l'expression de la réalité, comme le discours légitimé désignant des finalités auxquelles aspire le peuple tout entier:

C'est l'une des occultations communes du discours idéologique que de voiler cette exploration des fins et de prétendre que les fins proclamées ne sont que la volonté commune [...]. Or, précisément, cette volonté générale n'est pas un fait et c'est la première tâche des idéologues que de débattre de ces fins et d'amener une collectivité à approuver les fins et les moyens proposés (p. 109). ${ }^{5}$

\section{Fondements sociohistoriques}

Ne pouvant ici longuement développer les origines et les fondements sociohistoriques à la source de ces deux traditions, nous ne ferons que les esquisser en renvoyant le lecteur à diverses publications (Guiomar, 1990; Hobsbawn, 1992; Schnapper, 1994; Schulze, 1996; Thiesse, 1999). Le système éducatif formel et contemporain est une émanation de l'État-nation, unité politique aux formes très diverses, qui se met en place, à travers de nombreux conflits 6 , à partir des $17^{\mathrm{e}}$ et $18^{\mathrm{e}}$ siècles en Grande-Bretagne d'abord, puis aux États-Unis, en France ensuite et, enfin, en Allemagne et en Italie ${ }^{7}$, avant de se généraliser progressivement jusqu'au milieu du $20^{\mathrm{e}}$ siècle avec le processus d'indépendance des états coloniaux. L'État-nation, en tant que forme d'organisation moderne des sociétés occidentales, repose, d'une part, sur l'introduction dans le droit international du droit des peuples à disposer d'eux-mêmes, ce qui modifie radicalement les rapports entre nation et État ${ }^{8}$ et, d'autre part, sur l'idée d'individu dont Gourevitch (1997) présente l'émergence durant la période médiévale. Cet individu, dans l'État-nation, devient le citoyen, être politique et sujet abstrait libre et égal en droit (Schnapper \& Bachelier, 2000; Thériault, 1999).

Les deux traditions reposent à l'origine sur la même visée fondamentale, émancipatrice, de l'être humain. Fondamentalement, advenir être humain, en tant qu'individu libre, responsable et autonome, procède d'un processus d'émancipation. ${ }^{9}$ Telle est la visée éducative fondamentale dans tout État-nation démocratique! ${ }^{10}$

Pourquoi en est-il ainsi? Pourquoi cette idée d'émancipation est-elle au fondement de la mise en œuvre des systèmes modernes d'éducation? Parce que la finalité première de tout système d'éducation conçu dans le contexte d'un État-nation est de former des êtres humains émancipés, des individus ${ }^{11}$ égaux, libres et autonomes, ainsi que le prône n'importe quel État-nation. Sa spécificité est d'intégrer "les populations en une communauté de citoyens» (Schnapper, 1994, p. 28) et de leur garantir l'exercice de pratiques démocratiques, ce qui requiert la mise en place d'une «École démocratique [qui] doit donner à tous les capacités intellectuelles nécessaires pour participer réellement à la vie publique» (p. 95). 
Une telle finalité éducative requiert de passer par la culture, ce que, par exemple, Forquin (1989) et Williams (1965, 1968) montrent fort bien. Le concept de culture, sous l'impulsion allemande, entre autres des Fichte (1967), Schelling (1967), Schiller (1967), Schlegel (1841), Schleiermacher (1979) et von Humboldt (1979), devient dès lors central et porte une double signification. D'une part, il permet de fonder l'identité des citoyens d'un État-nation en cimentant, en unifiant le savoir. Dans la pensée allemande des $18^{\mathrm{e}}$ et $19^{\mathrm{e}}$ siècles la culture devient l'objet de la Wissenschaft, de l'étude philosophicoscientifique. D'autre part, la culture traduit le processus de développement du caractère, de la Bildung. L'université moderne rénovée est avant tout culturelle ${ }^{12}$ et elle s'exprimera pleinement en Angleterre avec le modèle libéral (Newman, 1925). Elle propose, relève Readings (1996), un modèle «qui vise à fusionner les traditions du passé aux ambitions futures au sein d'un champ unifié de culture» (p. 68). Elle trouve sa légitimité dans sa fonction primordiale de transmission de la culture et, dans le cadre des États-nations, de la culture nationale, en évitant, cependant, selon les conceptions de von Humboldt, de verser tant dans la seule réflexion philosophique en tant que loisir intellectuel que dans l'utilité pratique en tant qu'institution soumise aux volontés de l'État. La culture, ainsi comprise, est la réalisation émancipatrice de l'être humain par l'entremise d'un savoir ni indéterminé, ni déterminé dans son application: la culture est savoir vrai, libre, critique et unifié guidant l'action humaine; elle est source d'émancipation. Tant aux États-Unis qu'en France les systèmes éducatifs scolaires ont alors comme finalité première la formation d'un citoyen, c'est-à-dire le développement intégral de l'être humain cultivé: un être autonome, responsable, apte à agir dans la société de façon réfléchie et critique. Comme le note Goldring (2000) pour la France, "la mission de l'école n'est pas de remédier à l'inégalité sociale, mais de former des citoyens capables de penser par eux-mêmes pour fonder une société affranchie de l'ignorance et des liens serviles» (p. 9). ${ }^{13}$ Cependant, la mise en œuvre de cette visée repose, comme nous allons le voir, sur des traditions différentes, certains diraient sur des paradigmes différents.

\section{L'option française}

En France, dans sa loi d'orientation sur l'éducation du 10 juillet 1989 (Gouvernement de la France, 1989), loi qui a pour titre «Missions du système éducatif» et qui est rappelée dans les derniers programmes de l'école primaire (Gouvernement de la France, 1995), le ministère de l'Éducation nationale précisait dans le deuxième paragraphe que «l'école a pour rôle fondamental la transmission des connaissances» (p. 14). Il ajoutait dans un troisième paragraphe:

L'école a pour but de former, grâce à une réflexion sur ses objectifs pédagogiques et à leur renouvellement, les femmes et les hommes de demain, des femmes et des hommes en mesure de conduire leur vie personnelle civique et professionnelle en pleine responsabilité et capables d'adaptation, de créativité et de solidarité» (p. 14). 
Ainsi, le Gouvernement français rappelait la centralité historique de l'instruction dans le processus d'éducation scolaire. En effet, une telle orientation trouve ses origines dans la pensée d'acteurs de la révolution de 1789. Dans l'optique des révolutionnaires, l'atteinte de la liberté humaine passe prioritairement par l'instruction (l'acquisition du savoir). Cette conception résulte du développement de la pensée rationnelle cartésienne, de l'action des philosophes (entre autres Voltaire) et de celle des Encyclopédistes qui, au siècle des Lumières, se sont progressivement dressés contre l'obscurantisme, l'ignorance et l'oppression sociale qui caractérisaient, à leurs yeux, l'Église catholique, la royauté et l'aristocratie (Charlot, 1987; Éliard, 1994; Kintzler, 1989; Lelièvre, 1994; Léon, 1971). Dans l'esprit de la pensée républicaine, perçue comme la forme achevée de la société démocratique (Pettit, 1997), instruire est la responsabilité première de l'Étatnation, car elle est à la source de la liberté humaine et la meilleure assurance de former un citoyen libre.

Historiquement, la meilleure illustration de la position républicaine prônant un mouvement d'émancipation sociale vient sans doute des discours de Condorcet (1989a, 1989b, 1994) à l'Assemblée nationale en 1791 et 1792 . En 1791, Condorcet (1989a) écrit qu'il revient à la République d'assurer une éducation publique et que celle-ci «doit se borner à l'instruction» (p. 56), établissant ainsi une distinction majeure entre instruction et éducation, ainsi que le rappellent Lelièvre (1994) et Nique et Lelièvre (1993) qui confrontent les conceptions de Condorcet et de Ferry. ${ }^{14}$ L'école républicaine assure celle-là, celle-ci est affaire des parents et ne la regarde pas. En décembre 1792, dans un célèbre discours sur la question éducative à l'Assemblée nationale, il déclare que

former d'abord à la raison, instruire à n'écouter qu'elle, se défendre de l'enthousiasme qui pourrait l'égarer ou l'obscurcir, et se laisser entraîner ensuite à ce qu'elle approuve; telle est la marche que prescrit l'intérêt de l'humanité, et le principe sur lequel l'instruction publique doit être combinée. Il faut, sans doute, parler à l'imagination des enfants [...], mais il serait coupable de vouloir s'en emparer, même en faveur de ce qu'au fond de notre conscience nous croyons être la vérité (Condorcet, 1989b, p. 185).

Et de préciser:

L'éducation, si on la prend dans toute son étendue, ne se borne pas seulement à l'instruction positive, à l'enseignement des vérités de fait et de calcul, mais elle embrasse toutes les opinions politiques, morales ou religieuses. Or, la liberté de ces opinions ne serait qu'illusoire, si la société s'emparait des générations naissantes pour leur dicter ce qu'elles doivent croire. Celui qui entrant dans la société y porte des opinions que son éducation lui a données, n'est plus un homme libre; il est l'esclave de ses maîtres, et ses fers sont d'autant plus difficiles à rompre, que lui-même ne les sent pas, et qu'il croit obéir à la raison, quand il ne fait que se soumettre à celle d'un autre. [...] Les préjugés donnés par la puissance publique sont une véritable tyrannie, un attentat contre une des parties les plus précieuses de la liberté naturelle (Condorcet, 1989a, pp. 59-60). 
Critiquant l'Ancien régime bâti sur l'ignorance des masses, Condorcet préconise l'instruction perçue comme la voie à la liberté, car la connaissance ouvre la porte à la liberté. Le savoir est la fondation même du progrès, ainsi que Crampe-Casnabet (1985) le relève dans son analyse de l'auteur:

Tout homme a par nature droit au savoir, l'instruction doit être publique [...] il faut savoir lire, écrire, compter, connaître quelques principes fondamentaux des lois. Si chaque citoyen possède cette instruction élémentaire, l'érudition ${ }^{15}$ perd son pouvoir [...]. L'instruction a une finalité éminemment politique: sans instruction pas de liberté, pas d'égalité. La tyrannie est toujours liée à l'ignorance. L'instruction est à la fois le moyen du progrès des Lumières et le résultat de ce même progrès. Ainsi, c'est par le savoir que les hommes apprenant qu'ils ont des droits peuvent les faire progresser; c'est par lui que ce qui restait simple disposition inscrite dans la nature peut devenir réalité effective (pp. 63-64).

La notion d'instruction ne peut toutefois se réduire, dans la pensée de Condorcet, à la simple transmission de savoirs. L'instruction est libératrice et ne peut se concevoir sans l'appel à la raison, sans une démarche rationnelle. Le savoir, en tant que scientificité, se présente, selon cette logique, comme la médiation - cognitive - fondamentale du processus d'objectivation, d'appréhension du monde. Le rapport au monde exige de passer par un rapport au savoir. L'émancipation humaine réclame donc le recours au savoir scientifique et à la rationalité qui le sous-tend, car l'école républicaine française, selon la position mise en avant par Condorcet, rejette toute forme d'inculcation. Le plus sûr moyen de se prémunir contre tout danger d'influence idéologique (royaliste, religieuse, etc.) est la connaissance. Ainsi que le remarque Sachot (2000), la révolution française introduit une rupture majeure avec le système éducatif en vigueur jusqu'alors. Ainsi, le rapport au savoir, à la discipline scientifique, s'avère primordial, car il devient le garant de la tradition culturelle. Il importe donc de problématiser le savoir, d'en questionner le sens avant d'agir, de débattre des contenus cognitifs à soumettre aux apprentissages des futurs citoyens adultes: «En conséquence, le rapport au savoir est premier. Il importe dès lors, cependant, de se questionner sur ses attributs, sur sa scientificité, sur ses apports et de s'assurer que les savoirs sélectionnés sont à la fois exempts des biais idéologiques et qu'ils sont accessibles aux élèves. Les perspectives épistémologiques et didactiques viennent assurer ces fonctions». En conséquence, la recherche du sens et la définition des objets d'enseignement s'avèrent primordiales.

\section{Et aujourd'hui en France?}

Comme le souligne Vigarello (1994) «la République du XIXe siècle n’a pas concrétisé, loin s'en faut, le plan Condorcet» (p. 45). L'étude de l'évolution du système scolaire français montre plutôt l'importance de l'école de Jules Ferry (Éliard, 1994; Lelièvre, 1990, 1994; Nique \& Lelièvre, 1993; Prost, 1968), mais aussi sa mort au cours des années 1960 (Charlot, 1987; Prost, 1997). Nous attirons toutefois l'attention sur l'importance accordée aux contenus disciplinaires 
au sein des programmes d'études, importance qui s'est maintenue tout au long des $19^{\mathrm{e}}$ et $20^{\mathrm{e}}$ siècles. Kherroubi et Plaisance (2000) rappellent que «les instructions de 1969 recommandent au primaire de 'dépasser la notion contraignante de programmes'» (p. 28) et relèvent cet «ajout apparemment paradoxal de nouvelles disciplines» (p. 30), alors qu'un allègement des contenus est visé. Quant au secondaire, la place des disciplines scolaires demeure centrale dans la structure curriculaire.

Au regard de la formation à l'enseignement, si Zay (1994) fait état de trois modèles de formation - académique-disciplinaire, pédagogique, professionnel -, on observe aujourd'hui, à la suite de l'universitarisation et de la mise en œuvre de politiques de professionnalisation de la formation initiale, l'existence de deux modèles concurrents (le modèle professionnel ayant englobé le modèle pédagogique) pour concevoir la formation professionnelle des enseignants: le premier, au nom de la rationalité cognitive, mise sur l'acquisition préalable des savoirs de référence afin de guider l'action professorale; le second prône une centration sur l'action expérientielle, éclairée ensuite par une pensée réflexive (Chartier, 1998). Il s'agit là d'une confrontation plus large que de Queiroz (2000) qualifie de "frontière de feu» entre les tenants d'une conception de la transmission de savoirs disciplinaires et les défenseurs d'une conception "pédagogique», accusés de «détruire l'école républicaine» (Queiroz, 2000), entre, pour le dire bref, l'instruction et l'éducation ou, encore, entre une vision culturelle de l'école, lieu de formation désintéressée, et une vision professionnalisante, lieu de préparation à un emploi. Asher et Malet (1996) montrent la tension existant entre une tendance réformatrice qui entend lier davantage la formation à l'enseignement primaire et secondaire et le courant conservateur qui serait, selon leurs dires, porteur d'un «antipédagogisme latent». Ce courant rejette ce rapprochement, jugé impossible et impensable compte tenu de la nature et de la mission distincte des deux ordres d'enseignement. Il s'y oppose au nom du «principe séculier et universaliste de la transmission du savoir» (p. 276) et du statut républicain de l'école secondaire et de ses fondements culturels. ${ }^{16}$ Bulle (2000) souligne le dilemme entre l'attention portée «aux exigences cognitives des enseignements [... et] l'ouverture plus grande des systèmes éducatifs, qui n'est permise que par une dévalorisation de la valeur intrinsèque accordée aux savoirs transmis», ce qui a pour effet un "affaiblissement progressif de l'éducation cognitive assurée par les enseignements scolaires» (p. 371). Brucy et Ropé (2000) intitule d'ailleurs leur récent ouvrage Suffit-il de scolariser?, voulant par là signaler que "l'école est bien à la croisée de plusieurs enjeux: politiques, économiques, sociaux et culturels» (p. 207). Lang (1999) conclut pour sa part à un passage d'une définition de la professionnalité des enseignants du secondaire fondée sur des compétences culturelles et disciplinaires à une définition fondée sur des compétences professionnelles. Plus largement encore, Altet (1998) rappelle que «le ministère est passé d'un modèle de formation centré sur une logique d'enseignement et de maîtrise de contenus disciplinaires à une logique de qualité centrée sur la professionnalisation et d'ap- 
propriation de compétences professionnelles» (p. 72). Ce modèle "reste encore largement à construire» (p. 84) et Lacotte (Lacotte \& Lenoir, 1999) signale toujours une prédominance des enseignements disciplinaires dans les instituts universitaires de formation des maîtres (IUFM).

Enfin, s'il faut croire les chercheurs qui se sont penchés sur l'œuvre de Condorcet, on entend signaler l'actualité de sa pensée (Langouët, 1994), sa «modernité étonnante» (Terrot, 1997) et, en reprenant Éliard (1994), on peut souligner sa "passion extraordinaire pour l'émancipation de l'homme, pour l'abolition de toute forme de dépendance» (p. 64), finalité sociale que l'instruction peut promouvoir.

\section{L'option américaine}

Aux États-Unis, il en va autrement quant à l'actualisation des conceptions éducatives, encore que la visée soit la même. Marienstras (1988) rappelle que «la raison qui a conduit l'Amérique à l'indépendance - le droit à la liberté - est le principe suprême de la nation» (p. 405). Bacharan et Kaspi (2000) citent à ce propos la Déclaration d'indépendance rédigée par Jefferson: "Tous les hommes sont créés égaux. Ils sont dotés par le Créateur de droits inaliénables, parmi lesquels la vie, la liberté, et la recherche du bonheur» (p. 75). Ils ajoutent que ce dernier «avait défini ainsi la quintessence du rêve américain: devenir américain, c'est devenir un homme libre, c'est avoir toujours droit à une nouvelle chance» (p. 75). Reid (1998) illustre ainsi la conception américaine:

L'enseignement [...] doit être destiné: (a) à habiliter les étudiants à déterminer leur place et leurs possibilités dans la structure sociale et culturelle; (b) à comprendre le mouvement des idées anciennes et contemporaines qui ont façonné notre monde; (c) à comprendre les fondements du fonctionnement de notre système économique et de notre système politique; et (d) à saisir la différence entre des sociétés libres et répressives (National Commission on Excellence in Education, 1983, in Reid, 1998, p. 12).

Aux États-Unis, l'atteinte de la liberté humaine passe prioritairement par la socialisation, entendue ici comme le développement d'un savoir-agir en tant qu'intégration du savoir-faire et du savoir-être. Ce qui rend libre n'est pas directement lié aux connaissances, mais à la capacité d'agir dans et sur le monde. Éduquer revient alors à instrumenter dans un double sens, celui de la pratique et celui des relations humaines et sociales. C'est ainsi que se développe une conception "vocationaliste» axée, d'une part, sur le développement de symboles visant à concilier l'éthique protestante avec le nouvel ordre industriel et, d'autre part, sur le développement de formations professionnelles (Geoffroy \& Lenoir, 2000; Geoffroy, Lenoir \& Hasni, 1999). Les travaux de Kliebard (1986, 1992a, 1992b), de Rudolph (1977), de Tanner et Tanner (1990) montrent que cette conception résulte d'une préoccupation centrale pour l'insertion et l'intégration de l'être humain dans une société jeune, "ouverte», multiethnique, aux cultures et aux croyances religieuses diverses. 
Il importe essentiellement d'assurer alors la production de sujets humains, personnes individuelles, capables de participer harmonieusement aux activités sociales, politiques et économiques d'une société fondée sur le melting pot. ${ }^{17}$ De plus, les valeurs religieuses puritaines (du protestantisme) mettent en évidence l'importance du travail comme réalisation de soi et possibilité de plaire à Dieu. La nation américaine a établi par sa Constitution de 1787 et par ses amendements ultérieurs la séparation entre la religion et le politique. Cela n'en fait cependant pas une société laïque comme dans la République française. Alors qu'en France les idées démocratiques et l'esprit de liberté progressent en général contre l'Église, aux États-Unis, dès le départ, «la politique et la religion se trouvèrent d'accord" (de Tocqueville, 1968, p. 98). Le protestantisme, par sa diversité confessionnelle, ne peut faire la loi, ainsi que ce fut le cas dans les pays de religion catholique romaine - où, comme en France, le conflit, d'essence politique, s'est cristallisé autour de la laïcité (Charlot, 1987), dans un mouvement de sécularisation de la société qui s'inspire des principes universalistes républicains (Gautherin, 2000) -, mais il inspire fortement les mœurs: la religion garantit chez les Américains la conscience morale, le devoir civique, l'intériorisation de la loi. Et, en tant que jeune société qui entend rompre avec des modèles européens, jugés violents, inégalitaires, et considérés abusifs et obsolètes, il importait que les membres de la société bâtissent un «nouveau monde» qui repose sur des finalités et des valeurs sociales nouvelles. ${ }^{18}$ Ces deux dimensions idéologiques, celle de «destin manifeste» et celle de la religion comme garant moral, ont influé directement sur la conception de l'éducation: faire autrement qu'en Europe et lier l'éducation à une moralité issue de la religion protestante (Jacquin, Royot \& Whitfield, 2000; Marienstras, 1976, 1988).

Comme le montrent les travaux des quatre auteurs américains précités (Kliebard, Rudolph, Tanner \& Tanner), s'impose à la fin du $19^{\mathrm{e}}$ siècle, à la suite de la guerre civile et avec la vague de l'industrialisation galopante, un courant qui prône un renversement de conception de l'éducation et qui constitue, de la part de mouvements progressistes (Parker, Quincy, Ward, Herbartians, Dewey, etc.), une opposition aux conceptions humanistes traditionnelles mettant en avant une éducation non utilitariste, culturelle et académique (Rudolph, 1977, p. 14) qui découlait de la conception traditionnelle britannique, culturelle et humaniste, de l'éducation. Pinar (1998) l'énonce explicitement: «Les écoles publiques américaines ont été créées il y a plus de 100 ans pour préparer les citoyens à occuper des emplois dans une économie industrielle» (p. 205). C'est donc à cette époque et à la suite d'une maturation de quelque cent ans au cours desquels se forge l'État-nation étasunien que son système d'éducation intègre pleinement les attributs qui le fondent: une communauté d'individus citoyens unis par un ensemble de croyances et de symboles qui concourent au destin de l'État et qui sont orientés vers l'accomplissement de la fonction messianique de libération de tous les êtres humains par une action volontariste. Advenir américain ne se conçoit donc pas sans un comportement actif, par là sans une formation à l'agir, garant de la 
réalisation de la «destinée d'un peuple élu» et du «mythe américain» (Marienstras, 1988).

Pour Horkheimer (1974), le fondateur de l'École de Frankfurt, et pour ce dernier avec Adorno (1974), cette mutation se caractérise par le fait que la raison instrumentale, centrée sur l'immédiateté du présent, se substitue à la raison subjective des temps modernes, orientée vers l'avenir et le progrès, qui avait ellemême, antérieurement, pris le pas sur la raison objective, axée sur le passé comme système référentiel. Judy (1993) considère que la fondation de l'Université John Hopkins aux États-Unis en 1870 atteste pour la première fois de ce tournant crucial dans la conception de l'université en axant la formation supérieure vers la professionnalisation académique et l'enseignement d'un savoir instrumental. Ce nouveau modèle, dont Whitehead (1929) s'est fait le chantre, peut être qualifié de pragmatique, ainsi que le font Lessard et Tardif (à paraître) en reprenant en partie les travaux de Drèze et Debelle (1968) sur les conceptions de l'université.

Kliebard (1992a) explique comment, passant d'une conception mentaliste ${ }^{19}$ à la rationalité instrumentale, le principe de l'humanisme a été remplacé par celui du professionnalisme (vocationalism) dans la politique curriculaire des ÉtatsUnis. Théorie reliée de près au paradigme dominant du taylorisme - l'efficacité sociale (social efficiency) - et de son application dans le domaine de l'éducation par la Tyler rationale, celle-ci devient le guide principal des politiques et des pratiques curriculaires adoptées dans le pays. À cet égard, Withehead (1929) insiste pour que

la culture générale et la science se portent à la rencontre de l'action et participent au progrès de la société. Loin de considérer la culture et la science comme des fins en elles-mêmes, il n'a aucune objection à ce que l'éducation, quels que soient la forme et le contenu qu'elle prenne, soit utile: "Les pédants méprisent une éducation utile. Mais si l'éducation n'est pas utile, qu'est-elle? Est-ce un talent qu'il faut conserver secrètement dans une serviette? Bien sûr, l'éducation doit être utile, quel que soit votre but dans la vie. Elle le fut pour saint Augustin comme pour Napoléon. Et cela, parce que l'intelligence de la vie est utile» (in Drèze \& Debelle, 1968, p. 67) (Lessard \& Tardif, à paraître).

Ainsi, se tisse progressivement un lien étroit, sur le plan des finalités du système scolaire, entre une vision pragmatique, instrumentale (le savoir-faire) et une préoccupation pour l'insertion sociale, l'adhésion à des normes et à des valeurs qui caractérisent le peuple américain (le savoir-être). Selon Kliebard (1992a), la montée du professionnalisme s'est accompagnée de symboles servant à réconcilier l'éthique protestante du travail avec la nature du travail d'un nouvel ordre industriel en pleine expansion (dignité du travail versus dégradation du travail). De plus, Kliebard (1992a) soutient que le concept d'éducation fondamentale (general education) fut transformé radicalement au cours du premier quart du $20^{\mathrm{e}}$ siècle par les idées d'Herbert Spencer, principal exégète des conceptions de Charles Darwin appliquées à la société humaine et au domaine de l'éducation. 
Snedden (1921), lui-même disciple de Spencer, propose trois implications de la réforme spencérienne: un accent sur la science; un curriculum contrôlé par la loi naturelle; un curriculum peaufiné aux fonctions qui doivent être remplies pour survivre dans un monde moderne. On substitue ainsi la performance efficace des fonctions sociales à l'idée d'un curriculum visant le développement des ressources intellectuelles principales favorisant l'acquisition d'une culture humaniste.

Aux États-Unis, on ne pose donc pas le problème du savoir parce que ce n'est pas lui qui rend libre. Ce qui rend libre, c'est la capacité d'agir de façon autonome sur et dans le monde. La question centrale n'est pas celle du savoir, ni celle du sens, car le sens est donné par la société américaine, jugée a priori fondamentalement bonne et juste, puisque relevant d'un «destin manifeste» voulu par Dieu, mais celle de la fonctionnalité, du savoir-faire qui réclame un certain savoir-être. Le rapport au sujet y est donc premier. Il importe, dès lors, de se questionner sur les perspectives pédagogiques - le «comment faire» - qui favoriseront le mieux la mise en place de dispositifs appropriés pour atteindre ces finalités en permettant au sujet, d'une part, d'intégrer, à travers ses apprentissages, les normes et les valeurs sociales retenues au sein du curriculum, et, d'autre part, de développer les habiletés instrumentales requises pour intervenir sur et dans le monde.

\section{Et La formation à L'enseignement aux États-Unis?}

Sans nier l'existence de conceptions concourantes, ainsi que le montrent des auteurs comme Jackson (1992), Kliebard (1992b) et Liston et Zeichner (1991) ${ }^{20}$, la formation à l'enseignement aux États-Unis est marquée par une vision d'efficacité sociale. Consécutivement avant tout au choc de 1957 dû au lancement par les Soviétiques du premier Spoutnik et au sentiment concomitant que l'éducation scolaire frôlait l'échec complet (Barnabé, 1993), ce furent d'abord les ordres d'enseignement primaire et secondaire qui furent mis en cause. Puis, à la suite de nombreux rapports parus au tournant des années quatre-vingt (Liston \& Zeichner, 1991), dont $A$ nation at risk (National Commission on Excellence in Education, 1983), et avec la parution des rapports du Carnegie Task Force on the Education and the Economy Task Force on Teaching as a Profession (1986), du Holmes Group (1986, 1990, 1995) et de la National Commission on Teaching and America's Future (1996) ${ }^{21}$, c'est la formation à l'enseignement qui fut prise pour cible et qui se retrouva au cœur des débats. Toutes les critiques étaient dirigées vers la mauvaise qualité de la formation initiale à l'enseignement, considérée trop centrée sur l'instrumentalisation techniciste des futurs enseignants, inadéquate sur le plan des connaissances et, surtout, inappropriée par rapport aux besoins socio-économiques actuels, aux attentes de la population et aux caractéristiques de la clientèle. Le rapport de l'Education Commission of the States (1983), préoccupé par une éducation qui soutienne la croissance économique, réclame un engagement beaucoup plus important des États de l'Union et du monde des affaires dans la mise en place des réformes du système d'enseignement. 
Les réformes entreprises poursuivent essentiellement deux objectifs principaux: rehausser les standards de formation et la pratique et instaurer une formation professionnelle fondée sur un réel partenariat école-université. Un ensemble de mesures ont été adoptées: un retour aux apprentissages de base (back to basics), la valorisation des enseignements scientifiques et technologiques au détriment des perspectives humanistes (Carbonneau, 1993) ${ }^{22}$, l'introduction de standards de formation plus élevés 23 , la remise en cause de la formation de premier cycle universitaire (sous-graduée) jugée insuffisante (Clifford \& Guthrie, 1988; Goodlad, 1990a), la mise en œuvre d'une formation professionnelle qui devrait se modeler sur la formation médicale (Holmes Group, 1990; Reed, 1993), une professionnalisation du corps enseignant impliquant une responsabilisation de leur pratique (Abbott, 1991), une accréditation des institutions de formation (Parker, 1993) et une évaluation externe régulière de la pratique professionnelle, l'instauration de professional development schools, la promotion d'approches favorisant l'établissement de communautés d'apprentissage en classe et avec le milieu social environnant (Raymond \& Lenoir, 1998), le développement d'un savoir professionnel de base (pedagogical content knowledge), la professionnalisation des formateurs universitaires (Labaree, 1992; Holmes Group, 1986) et une reconceptualisation des modèles de formation (American Council on Education, 1999), etc.

La vision qui ressort de toutes ces propositions et mesures, évidemment plus ou moins appliquées, entre autres parce que l'éducation scolaire est décentralisée et relève de chaque État de l'Union (Lessard, Lenoir, Martin, Tardif \& Voyer, 1999), conduit Pinar (1998) à voir dans ces réformes le passage du modèle de l'école manufacture - qu'il qualifie aussi de modèle de la ligne d'assemblage - au modèle de la corporation, modèle systémique plus souple vis-à-vis de la structuration curriculaire et de l'organisation pédagogique, et plus ouvert aux différences du contexte social et de la clientèle étudiante. Pinar souligne que ces changements ne mettent nullement en cause les finalités éducatives, parce que «l'organisation et la culture de l'école sont liées à l'économie» (p. 206).

\section{Quelques impacts et enjeux au regard de la formation à l'enseignement}

Pour notre part, en continuité avec cette interprétation de Pinar et sans nier l'existence d'apports tout à fait pertinents en ce qui regarde la formation à l'enseignement aux États-Unis, ainsi que le notent avec certaines nuances cet auteur ou encore Judge (1998), nous formulons l'hypothèse que cette tendance s'inscrit dans le courant néolibéral qui soutient actuellement le phénomène de mondialisation - économique, politique, symbolique, culturelle - en cours (Aronowitz, 2000; Burbules \& Torres, 2000a; Freitag \& Pineault, 1999; Gélinas, 2000; Mercure, 2001). Cette idéologie, appliquée à l'éducation scolaire, prend pour mo- 
dèle l'entreprise multinationale, la corporation, prototype des structures organisationnelles auxquelles doivent se soumettre les différentes institutions sociales.

\section{Vers une éducation de L'excellence}

Ainsi que nous le rappelons (Lenoir, 2000a, 2000b, à paraitre) plusieurs auteurs mettent en évidence différentes caractéristiques qui sont en train d'imprégner progressivement les systèmes scolaires occidentaux et qui conduisent l'éducation, soumise à la logique de l'économie capitaliste de marché, à devenir un processus marchand. C'est ainsi que Petrella (2000) considère que l'éducation fait face à cinq pièges qui tendent à la détourner des dimensions spécifiquement humaines.

Selon différents spécialistes de la question au Québec, dont Beauchemin, Bourque, Duchastel, Boismenu et Noël (1995) et Readings (1996), pour répondre aux exigences de ce phénomène global de mondialisation, le discours néolibéral met en avant le principe d'excellence, et il l'accompagne d'autres termes - la privatisation, le rendement, l'efficience, l'efficacité, la performance, les compétences, la flexibilité, l'imputation, la responsabilisation, la gestion de la qualité, la reddition de comptes - en vue d'harmoniser les pratiques sociales avec les impératifs du nouvel échiquier mondial où semble se jouer dorénavant le destin des sociétés. La libéralisation des règles du marché est en train de créer «un espace unique mondial où circulent librement marchandises, capitaux, services et personnes dans une logique de compétitivité universelle» (Fournier, 1997, p. 46). Et cette logique, constate Petrella (1995), concerne «tous les hommes, tous les groupes sociaux institués, toutes les communautés territoriales (villes, régions, États)» (p. 28). Le système scolaire ne pouvait évidemment échapper à cette tendance forte. Comme le relèvent Burbules et Torres (2000b), «la version néolibérale de la globalisation [...] se reflète en éducation en privilégiant, sinon en imposant des politiques particulières sur le plan de l'évaluation, du financement, des normes, de la formation des enseignants, du curriculum, de l'instruction et des examens» (p. 15).

Ainsi, cette nouvelle conception de l'école, dont la formation à l'enseignement pourrait (devrait?) devenir un des fers de lance en vue d'établir et de conforter la "société du savoir», vise à assurer la flexibilité de l'enseignement postsecondaire pour répondre rapidement aux besoins de l'entreprise et à réformer l'enseignement primaire et secondaire pour assurer le développement des habiletés et des compétences requises par des travailleurs vivant dans un monde global (acquisition des savoirs essentiels, dits fondamentaux; inculcation de qualités telles que la confiance en soi, les qualifications et les connaissances requises pour l'obtention d'un emploi; développement de l'aptitude à vivre dans une société multiethnique et multiculturelle; etc.). D'où l'adoption généralisée de l'approche par compétences, originellement développée dans le milieu de l'industrie (Tanguy, 1994). Popkewitz (2000) remarque que «dans de nombreux pays, les réformes curriculaires sont concernées moins par les contenus spécifiques des matières scolaires que par la production d'un être humain qui puisse «se sentir 
"chez lui» avec une identité cosmopolite qui incorpore une flexibilité pragmatique et des dispositions à résoudre des problèmes» (p. 171). Martinelli, dès 1979, mais aussi, par exemple, Aronowitz (2000) et Readings (1996) montrent bien que le contrôle des processus socioculturels constitue un mécanisme fondamental dans le développement actuel du modèle néolibéral au sein du processus de mondialisation.

Cette nouvelle conception vise aussi à assurer une "obligation de résultats», d'où l'idée d'excellence ainsi que les contrats de performance et les indicateurs de qualité de l'enseignement qui reflètent l'assimilation de la notion d'école à celle d'usine en adoptant la logique comptable qui prévaut dans l'entreprise (rapport "coût-productivité-rentabilité») (Lenoir, à paraître). Toujours selon cette orientation, il importe de gérer la clientèle scolaire en tant que consommatrice de savoirs et de la transformer en "capital humain" prêt à fonctionner à la sortie (une "pédagogie du service»), ce qui entraîne l'institution scolaire à considérer de moins en moins les dimensions humaines, sociales et culturelles, mais à éduquer pour la ressource humaine et non plus pour et par la personne humaine. Il importe également de développer une éthique de la responsabilité individuelle (selfmanagement des écoles) axée sur les valeurs entrepreneuriales en mettant en avant la socialisation en tant qu'insertion "harmonieuse» dans la société (Lenoir, sous presse).

Bref, l'objectif est de subordonner les processus éducatifs aux «attentes sociales» que sont la privatisation, la déréglementation, la redéfinition des rapports entre privé et public, l'approfondissement de l'individualisme, l'extension d'une culture de masse standardisée et consommable, l'application de la rationalité économique comme norme universelle, etc. Ce qui semble alors fonder en dernier recours «l'excellence» universitaire, ce serait sa capacité de performer dans un marché en expansion. L'université devient alors une organisation bureaucratique, technocratisée selon Freitag $(1995,1996)$, qui ne peut plus d'aucune façon être considérée comme un appareil idéologique d'État (Althusser, 1976); bien au contraire, elle s'insère dans le réseau organisationnel mondial et elle devient une organisation toute tendue vers la recherche de l'excellence qui, à bien y réfléchir, peut vouloir dire «recherche de productivité», économique s'entend, dans la mesure où l'université est dorénavant assimilée à une entreprise qui vend des services. Mais cette finalité, qui sert également de référent et de critère premier de mesure, manque dramatiquement de contenu humain de formation, de perspectives sociales, de la portée "civilisationnelle» qui caractérisait l'université depuis sa fondation il y a quelque 800 ans, ainsi que le met en relief Freitag (1995).

La conception pragmatique jusqualors prédominante en éducation aux États-Unis se transforme en profondeur. Le nouveau modèle qui, aujourd'hui, est parti à la conquête du monde, porté par une mondialisation en plein essor, se caractérise, si l'on veut bien suivre la pensée de Readings (1996), par l'adhésion à un slogan, l'excellence. Si le mot peut incorporer toutes les significations qu'on voudra bien lui accorder, pour Readings, «la notion d'excellence, qui fonctionne 
moins pour permettre la mise en place d'observations effectives que pour assurer une évaluation exhaustive, sert bien davantage à insérer l'université dans un filet d'institutions bureaucratiques» (p. 29), en fonction des besoins de la mondialisation des marchés et des intérêts des grandes firmes transnationales. L'idée d'excellence élimine toute référence aux conceptions antérieures, que celles-ci soient portées par l'un ou l'autre des modèles culturaliste ou scientifique qui ont prévalu antérieurement dans le monde universitaire. Elle rompt ainsi également le lien traditionnellement existant entre la fonction d'acculturation nationale assumée par l'université et la formation d'un peuple républicain citoyen d'un État-nation. L'université de l'excellence serait-elle alors une organisation transnationale en devenir au service, non du peuple, mais des intérêts des grandes corporations qui visent à contrôler l'économie mondiale, à produire une culture de masse standardisée et consommable, et à contrôler les orientations politiques des États-nations? Il ne s'agirait pas seulement d'un désengagement de l'État, mais bien d'un recul de l'idéal démocratique (Pena-Ruiz, 2001; Saltman, 2000), dû à la faiblesse du contrôle politique, du fait que les corporations transnationales, qui dictent de plus en plus les règles du jeu et qui ne sont guère préoccupées par les principes démocratiques, échappent au pouvoirs étatiques nationaux (Robinson, 1995), du fait également que ces pouvoirs nationaux, soumis de plus en plus aux mécanismes du marché international, se voient progressivement affaiblis et grugés. L'autorité politique, de plus en plus assujettie aux grandes firmes transnationales (systèmes d'entreprises et systèmes financiers), se déplace en outre vers des entités supranationales (l'Alena, la Communauté européenne, etc.) et internationales (Le FMI, la Banque mondiale, l'ONU, etc.), entités qui imposent aux États-nations «les diktats des bureaucraties supranationales» (Thériault, 1997, p. 29).

\section{Vers une excellente formation à l'enseignement ou vers une formation à l'enseignement de l'excellence?}

Les réformes actuelles de la formation à l'enseignement, aujourd'hui universitarisée en France comme aux États-Unis, ont pour raison officielle d'améliorer la préparation des futurs enseignants et la qualité de leur intervention éducative. Sachant que près de deux millions et demi de nouveaux enseignants devront être engagés aux États-Unis au cours de cette décennie (American Council on Education, 1999), il s'agit là indubitablement d'un enjeu majeur. Le remplacement du corps enseignant français sera également considérable puisque plus de $40 \%$ des enseignants quitteront au cours des dix prochaines années (Lang, 2001). Par ailleurs, si on se questionne sur les effets de l'enseignement sur les apprentissages et si, en fait, on en sait relativement peu à cet égard (Bressoux, 2002; Bru, 1991, 1997), l'American Council on Education (1999) juge cependant que «les enseignants exercent une influence forte et remarquable sur la performance académique des élèves» ( $p$. 3). La qualité de la formation à l'enseignement s'avère dès lors cruciale.

Or, sans vouloir remettre en cause le bien-fondé des différents concepts qui animent ces réformes (approche programme - c'est-à-dire approche curriculaire 
intégrée -, pédagogie par projets, approche par problèmes, perspectives interdisciplinaires et transversales, approche par compétences, développement professionnel, mise en place de communautés apprenantes, etc.) ${ }^{24}$ et qui sont éventuellement garants de profondes modifications dans les pratiques enseignantes, une distanciation critique, alimentée par les différences de tradition en éducation et par les orientations socio-idéologiques néolibérales qui imprègnent le phénomène de mondialisation, s'avère nécessaire. Considérons la formation à l'enseignement sous ces deux angles d'approche critique.

\section{Des perspectives socio-historiques distinctes}

À comparer les systèmes d'actualisation de l'éducation en France et aux ÉtatsUnis, on voit bien l'existence de perspectives socio-historiques distinctes. Dans le monde anglophone des États-Unis, le savoir-faire et le savoir-être sont premiers, car, selon Reid (1998),

le monde que l'éducation voudrait aider à créer est présenté comme objectif, alors qu'il paraît subjectif pour les étudiants européens. Pour l'étudiant américain, [...], le monde social et culturel est une structure objective qui se compose de systèmes (comme le système économique ou le système politique). La tâche du curriculum est alors de présenter aux étudiants cette structure et de les aider à déterminer quelle place ils occuperont en son sein. Les prémisses en arrière d'un tel raisonnement sont, premièrement, que la culture et la société peuvent être rendus par des faits qu'il faut apprendre et, deuxièmement, que, pour les étudiants, la question de la façon avec laquelle ils tisseront des relations avec la société et la culture dépend de la réponse qu'ils voudront bien faire, car ils demeurent complètement libres. Le seul point où les valeurs entrent ouvertement dans les prescriptions réside dans la référence aux sociétés "libres» et "répressives». Et, sur ce point, il ne peut y avoir ni ombre ni gris. Les sociétés tombent apparemment clairement dans l'une ou l'autre de ces catégories ${ }^{25}$. C'est cela aussi qu'ils doivent apprendre (p. 13).

Il importe, dès lors, de se questionner sur les modalités à mettre en œuvre pour permettre à des sujets individuels de s'intégrer à la société américaine, société "objective» et «libre» par excellence, en fonction de ses valeurs (la démocratie) et de son mode de vie. L'émancipation humaine qu'offre la société démocratique américaine exigeait donc jusqu'à tout récemment le développement d'une approche pragmatique - c'est-à-dire de pratiques tant sociales qu'instrumentales qui repose sur une inculcation de quelques principes et valeurs fondamentales pouvant garantir l'identité citoyenne et conforter l'idée de la supériorité nationale. Cette approche est certes maintenue, mais aujourd'hui «enrichie» par la notion d'excellence. Efficience et efficacité dans un système hautement compétitif sont devenus, parmi d'autres, des maîtres-mots.

On comprend bien dès lors que les préoccupations qui ont animé les curriculums et les pratiques de formation à l'enseignement aux États-Unis ont porté avant tout sur la mise en œuvre des perspectives pédagogiques et sur les processus d'apprentissage qu'elles pouvaient favoriser. C'est ainsi que la déclaration, in- 
troduite officiellement en France en 1989, que l'élève est au centre des apprentissages, a cours depuis une centaine d'années dans le discours éducatif étasunien. La conception française était différente, en ce qu'elle reposait, nous l'avons précédemment rappelé en nous appuyant sur plusieurs auteurs français, sur une préoccupation pour le développement d'une rationalité cognitive fondée sur les savoirs scientifiques et problématisant le social. Le Rapport de la Commission de réflexion sur les sciences de l'éducation (CORESE), rédigé sous la responsabilité de Charlot (1995), témoigne bien de l'ancrage profond du système des disciplines dans la conception de ce que doit être un processus de formation à l'enseignement en France. Il illustre tout particulièrement la tension qui règne entre les sciences de l'éducation assurant la charpente de cette formation et une formation professionnalisante. Se penchant sur la formation universitaire, la Commission privilégie nettement un cursus de type disciplinaire, même quand elle aborde la question de la professionnalisation, estimant que les sciences de l'éducation contribuent déjà de façon significative à la professionnalité tout en constatant que la pluridisciplinarité qui caractérise les formations professionnelles empêche de clairement identifier leur apport spécifique à ces formations et que les étudiants qui suivent ces formation sont, du moins en partie, déjà professionnalisés. Ainsi que nous l'avons signalé au début de cet article, pour Lang (1999) le mouvement de professionnalisation et la création des IUFM ont un ensemble d'effets qui ébranlent fortement la logique traditionnelle. Le statut social et l'identité professionnelle, qui reposaient sur une culture faite de hauts savoirs, se voient menacés entre autres par une restructuration de la formation à l'enseignement, par l'introduction d'autres façons de concevoir l'apprentissage et par le déplacement de la transmission d'un patrimoine culturel à une adaptation aux exigences de la vie contemporaine.

Nous formulons alors l'hypothèse que la différence de centration, sur le savoir - le «pôle-objet» - en France républicaine et sur la personne en situation d'apprentissage - le «pôle-sujet» - aux États-Unis «s'expliquerait dans la reconnaissance en France d'un construit social constitutif de l'être éduqué (le patrimoine culturel), construit à enseigner aux futurs citoyens, alors qu'aux États-Unis, on fait face à un construit social à constituer pour éduquer le futur citoyen, ce qui explique la centration sur le "pôle-sujet»» (Lenoir, 1999, p. 310). C’est pourquoi, dans l'enseignement et dans la formation à l'enseignement, «la priorité serait donnée en France à la transmission valorisée du savoir entendu comme patrimoine culturel, tandis qu'aux États-Unis, elle serait accordée à l'adhésion aux «vertus» et aux valeurs de la société américaine, au savoir-être, ainsi qu'au savoir-faire axé sur la résolution de problèmes» (p. 310). Dans ce sens, si les deux systèmes d'éducation ont fait appel au savoir-être, à la socialisation (en France, avec Jules Ferry tout particulièrement), elle n'est aucunement fondée sur les mêmes principes car la conception de la socialisation ne repose pas sur le même modèle de citoyenneté.

Par ailleurs, des deux côté de l'Atlantique, les systèmes scolaires recourent largement aux mêmes termes. Il importe toutefois de ne pas se laisser mystifier par 
cet usage commun. Cela ne signifie nullement qu'ils sont porteurs des mêmes significations et qu'ils s'intègrent à des trames conceptuelles identiques. À titre illustratif, Sachot (2000) le montre bien pour le concept de curriculum qui se trouve peu à peu introduit dans le vocabulaire éducatif français. Ce concept, selon cet auteur, demeure étranger aux principes républicains et, au-delà, au concept de discipline scientifique entendu comme problématisation du savoir. Il est même, pour Sachot, un contre-modèle de la discipline:

La discipline a pour fonction première d'instruire un questionnement de la personne quant au vrai, de manière qu'elle puisse juger et décider "en connaissance de cause» [...]. La logique curriculaire n'est pas républicaine, au sens où par République on n'entend moins un régime politique spécifique que l'assemblée des seuls individus qui la composent, la seule instance détentrice de la souveraineté et instituante des formes constitutives de l'organisation en société, que ces formes soient politiques (patrie, État, nation...) ou économiques (sociétés financières ou de production), religieuses, associatives, etc., autrement dit, l'instance qui soumet toutes ces instances internes aux principes que la seule raison - et non l'intérêt impose et qui sont de soi universels. Le curriculum se définit à partir des attentes et des besoins de la société, telle qu'elle est dans sa diversité et ses changements (p. 25).

\section{Le poids de la mondialisation et de l'idéologie néolibérale}

Dès lors, de deux choses l'une: ou bien ce concept se voit chargé d'un sens distinct, ou bien il témoigne d'une transformation des éléments constitutifs de la République et de la transformation du rapport au savoir et, plus profondément encore, de la transformation de l'institution scolaire. Sachot penche vers cette deuxième hypothèse. Les idées circulent aujourd'hui partout dans le monde, se rencontrent, se heurtent, se compénètrent, certaines imposent leur loi. Les conditions mondiales font que les deux traditions éducatives sont aujourd'hui profondément perturbées par le processus de mondialisation en cours et par l'emprise hégémonique qu'opère sur elle l'idéologie néolibérale, couplée à une gouvernementalité de plus en plus économique, qui échappe peu à peu aux pouvoirs politiques et qui tend à l'autonomisation du capital financier (Chesnais, 1997), le capitalisme politique devant bientôt s'afficher dans les pages nécrologiques (Freitag \& Pineault, 1999). Aux États-Unis cependant, le changement semble plutôt s'effectuer dans la continuité et non selon une certaine rupture comme en France, ce dont atteste le développement, dans le champ de l'éducation, de la notion d'excellence ${ }^{26}$ et des autres concepts qui lui sont associés.

Enfin, le phénomène d'internationalisation qui favorise actuellement la rencontre des organismes, des personnes et des idées, laisse voir l'existence d'une convergence grandissante entre les deux traditions. Un "processus de "glocalisation", c'est-à-dire l'inscription au plan local de modes de pensée et d'expression qui circulent dans l'espace-monde» est en train de se répandre, observe Novoa (2001, p. 104). Le questionnement sur les disciplines va croissant aux États- 
Unis, nous l'avons signalé, et le rôle de l'enseignant y devient une préoccupation accrue. En France, la tradition qui soutenait les modèles antérieurs en place se voit ébranlée par les pressions des pouvoirs économiques et par l'influence économique, politique, culturelle et idéologique des États-Unis qui distille peu à peu à travers la planète ses différents modèles. Le conservatisme des pratiques enseignantes est de plus en plus questionné, ne serait-ce qu'à cause d'un taux d'échec considéré aujourd'hui socialement inacceptable, et on accorde plus d'attention aux questions pédagogiques, aux stratégies d'enseignement, à la gestion de la classe. Il semble évident, comme le remarquent Hopmann et Gundem (1998) et Hopmann et Riquarts (1995), qu'un dialogue est amorcé entre les deux traditions, qu'ils identifient comme la tradition curriculaire et la tradition didactique.

Mais s'agit-il bien d'un dialogue? Ou, plus exactement, d'une invasion tranquille, insidieuse, qui peu à peu s'immisce au sein de la logique française et y provoque des tensions? Est-ce un bien pour la formation des enseignants en France? Quel jugement, quelle évaluation porter face à ce dialogue ou à cette intrusion? Là n'était pas l'objet de ce texte. Nous constatons le phénomène et nous cherchons à le démonter pour mettre en exergue quelques-unes de ses composantes. Il ne s'agit donc nullement d'exprimer une quelconque opposition de principe vis-à-vis des deux traditions exposées et de leurs modes d'actualisation, en cohérence dans leur constitution initiale avec leurs visées, encore moins de mettre en cause le processus de professionnalisation des métiers de l'enseignement. Là où nous introduisons des réserves, c'est dans l'interférence que l'idéologie néolibérale joue dans la mise en place de nouveaux systèmes d'enseignement, de nouvelles valeurs éducatives, de nouveaux modèles d'enseignement, car cette idéologie n'a que faire des principes démocratiques qui ont animé la vie sociale et politique des deux derniers siècles, et elle est source de multiples processus d'exclusion sociale que soutiennent entre autres les mouvements de privatisation (Carnoy, 2000; Whitty \& Edwards, 1998), la marchandisation de l'école allant en se développant avec celle de l'individu qui, en contre-coup, se réfugie dans un individualisme exacerbé et dans une citoyenneté particulariste. Ainsi, l'individualisation accrue et une mondialisation investie de l'idéologie néolibérale combinent une fuite vers le bas (vers l'individu se repliant sur soi et se réfugiant dans différentes formes de "tribalisme») et une fuite vers le haut (vers la rationalité technique, instrumentale, liée à la mondialisation), ce qui entraîne la société globale, qui constituait le ciment intégrateur des États-nations (Bourque, Duchastel \& Kuzminski, 1997; Fournier, 1997; Tremblay, 1997), à se dilater par les deux bouts, ce qui la conduit à l'éclatement par la suppression de la médiation (la nation) qui liait le corps social et le corps politique. Ces dispositions ne sont pas sans susciter de sérieuses inquiétudes au regard de la formation à l'enseignement. 


\section{Conclusion}

Ces tendances, fréquemment dénoncées depuis quelques années, ont été récemment synthétisées par le président de l'Unesco:

Les risques que présente un tel phénomène sont multiples et largement débattus: uniformisation, standardisation des messages, mercantilisation du patrimoine, des biens et services culturels. Mais aussi perte des identités, appauvrissement de la pensée, traçant ainsi la voie à un repli sur soi défensif, à l'exacerbation des particularismes et à la montée des intolérances. Du point de vue des libertés, les dangers ne sont pas moins redoutables: celui des nouveaux rapports de domination économiques, réduisant à l'impuissance les pouvoirs démocratiques. [...] La nécessité de la sauvegarde de la "diversité culturelle» est probablement une autre de ces références de plus en plus largement partagée. Et à juste titre. Car la "diversité culturelle» est tout aussi fondamentale à la survie de l'humanité que la "diversité naturelle» (Matsuura, 2000, pp. 2-3).

Le philosophe Walzer (1997), traitant d'éducation, écrit: «J'ai le sentiment que toute école préparatoire, conçue comme une entreprise commerciale, sera un instrument de tyrannie» (p. 299). Faudrait-il que la formation à l'enseignement soit prochainement conçue Sous le soleil de Big Brother (Brune, 2000)?

Plus généralement, l'analyse comparative des deux traditions nous conduit à penser qu'elles traduisent une tension qui résulte des finalités et de la fonction de tout système scolaire moderne. En s'inspirant de Bourdieu (1967) qui distingue entre des fonctions internes, de conservation culturelle, et des fonctions externes, d'adaptation sociale, la raison d'être d'un système éducatif émanant d'un Étatnation étant, sur le plan idéologico-discursif du moins, ainsi que nous l'avons rappelé, l'émancipation des êtres humains futurs citoyens, deux approches ressortent d'une part celle qui passe par l'instruction ou par l'instrumentalisation, en lien avec les fonction externes qui expriment, selon Bourdieu, une tendance à l'inertie (un système ne se modifie que contraint, forcé, en cherchant à résister aux changements qui lui sont imposés), et, d'autre part, celle qui s'appuie sur la socialisation, en lien avec les fonctions internes qui expriment une tendance à l'autonomie (son propre univers de fonctionnement avec ses caractéristiques). Ces différentes modalités opérationnelles, qui se compénètrent, se chevauchent, se rejettent, sont alors à la source d'une tension dans le rapport qui s'établit entre les êtres humains, sujets et acteurs sociaux, et la société.

Nous tendons donc à appréhender les tensions et les débats qui ont cours dans la formation à l'enseignement, tout particulièrement entre une centration sur l'enseignement des savoirs, liée au développement d'une identité disciplinaire, et une centration sur les pratiques d'enseignement, liée au développement d'une identité professionnelle, sous l'éclairage de l'idéologie néolibérale et des changement sociaux que suscite le processus de mondialisation et l'internationalisation des échanges. Plus profondément, les orientations qui s'imposent de nos jours en éducation et en formation à l'enseignement vont-elles aller dans le sens 
d'une réelle élévation des compétences professionnelles riches, d'une excellente préparation à l'enseignement, ce dont doute par exemple Pinar (1998), ou dans le sens d'une préparation ayant pour visée éducative un enseignement de l'excellence? Ou plutôt dans le sens des deux à différentes doses selon les sociétés...

En conclusion aux perspectives que nous venons de présenter, une des prochaines questions sera peut-être de se demander jusqu'à quand les États-nations garderont une prise effective sur leur système d'enseignement. Dit autrement, quand, comment et jusqu'à quel point le contrôle des ordres d'enseignement primaire et secondaire passera entre les mains de la sphère économique, considérant que cette transition est déjà fort avancée pour les universités, aux États-Unis du moins?

\section{Notes}

1 Si quelques écrits sont parus en anglais aux États-Unis sur le concept de didactique (par exemple, Hopmann \& Gundem, 1998; Hopmann \& Riquarts, 1995; Tochon, 1999), aucun ne provient, à notre connaissance, de la plume d'auteurs américains. Dans les publications où des auteurs américains ont rédigé des chapitres, ceux-ci portent exclusivement sur le concept de curriculum.

2 Est didactique, pour un anglophone, une pratique d'enseignement surannée et hautement ennuyeuse de surcroît.

3 Aux États-Unis, une des questions qui agitent de plus en plus le monde de l'enseignement porte sur l'éventualité de prendre davantage en compte les savoirs des enseignants dans le cadre de leur développement professionnel (Tillema, 1995; Vosniadou, Ioannides, Dimitrakopoulou \& Papademetriou, 2001; Wilson \& Berne, 1999). Les travaux de Doyle (1990) sur les relations entre contenu et gestion de la classe, et ceux de Shulman (1987) et Gudmundsdottir (1988) sur le pedagogical content knowledge, par exemple, avaient déjà attiré l'attention sur l'importance des savoirs à enseigner dans le cadre d'une formation initiale à l'enseignement. Notons que le pedagogical content knowledge inclut d'autres savoirs que les savoirs disciplinaires. C'est dire que leur importance n'a qu'une place relative parmi les autres préoccupations qui marquent l'univers de l'enseignement et de la formation à l'enseignement aux États-Unis.

4 Cet article découle en grande partie des notes d'un cours dispensé en février 2001, à titre de professeur invité, à la Faculté des sciences psychologiques et de l'éducation de l'Université libre de Bruxelles (Lenoir, 2001a). Nous tenons à remercier tout particulièrement l'un des lecteurs de la version initialement soumise pour les commentaires aussi abondants que riches et détaillés qui nous ont guidé dans la rédaction de la présente version.

5 Pour Ansart (1974), à la différence du système de pouvoir, «action sur autrui [...] obtenue par l'obéissance et par la menace des sanctions de force, le système idéologique, au contraire, obtient la répercussion sur les comportements par le maniement des significations» (p. 56). Il "proclame le sens, c'est-à-dire le sens vécu de la vie collective qui permettra de faire de l'action commune une vérité et de transposer chaque pratique dans l'ordre de l'intelligible et de la raison» (p. 15) et il «ne revêt ses effets que dans la mesure où les messages sont reçus dans le consentement et sont vécus comme évidences par les consciences individuelles» (p. 81). Par ailleurs, Ansart signale la nécessité d'un processus de discrédit en tant qu'un des processus indispensables auquel doit recourir une idéologie en élaboration: "Quant à la création idéologique, elle s'opère à partir du champ constitué par un double travail de critique à l'égard des systèmes présents et de proposition d'une syn- 
thèse nouvelle» (p. 77). C'est bien, par exemple, ce qui peut être observé de la part de l'idéologie néolibérale (Anderson, 1996; Dostaler, 2001; Klein, Tremblay \& Dionne, 1997; Pasche \& Peters, 1997), à la suite de la proclamation de la «fin des idéologies» (Bell, 1967), proclamation fondamentalement idéologique qui lui permet de se présenter comme un ordre nouveau et providentiel, fondé sur l'analyse scientifique et la prise en compte des faits empiriques émanant du réel. De plus, toujours en suivant Ansart (1974), rappelons que «le discours idéologique est discours de légitimation. Il s'agit, en disant les raisons d'être d'une organisation, d'en démontrer la valeur éminente, la conformité à la justice. Pour y parvenir, le discours fait communément appel à un "fondement» tenu pour absolu et d'autant plus incontestable qu'il est hors de portée de la vérification [...]. L'organisation proposée n'est pas un ordre accidentel issu du hasard des circonstances ou des déraisons individuelles, elle répond à un principe ou à une nécessité qui la rend indiscutable» (pp. 17-18).

6 Il faut se rappeler les guerres civiles françaises du 16e siècle et l'État absolu de Louis XIV, les guerres de religion, les guerres d'expansion territoriale, les révolutions sociales ou politiques qui ont eu cours en Europe à partir de 1500.

7 En Allemagne (comme en Italie) se maintient le lien entre ethnicité et nation qui oppose ainsi l'État-nation (Staatsnation) et la nation culturelle (Kulturnation), la nation étant vue en des termes organicistes à partir de l'idée de communauté d'un peuple originel (Urvolk), issu d'une même ascendance, partageant une même culture et un même passé (un peuple, une langue, une culture). Cette conception repose sur les travaux de l'École du droit historique qui, à l'encontre de celle du droit naturel, recourt à l'idée de communauté concrète (un peuple), d'un corps social régi par des liens établis par la coutume (la langue, la culture). Elle est à l'origine de la notion de nationalité fondée sur l'idée de pacte ethnique (et non de pacte social comme en France).

8 Voir les travaux de l'École moderne du droit naturel au $18^{\mathrm{e}}$ siècle qui s'appuie sur l'idée de droits universels et individuels, mais impersonnels.

9 Nous nous plaçons ici d'un point de vue historique strictement occidental.

10 En fait, au-delà du discours, le tout est de savoir si la finalité de l'éducation est l'émancipation humaine ou une des formes quelconques, subtiles ou non, d'asservissement, de soumission sociale à une idéologie hégémonique quelconque, à l'utilitarisme ou à un extrémisme religieux par exemple, et à des pouvoirs particuliers. Nous voulons également souligner que si nous insistons sur l'émancipation comme finalité de l'éducation scolaire, c'est pour mettre en évidence le projet historiquement conçu par les réformateurs qui ont pensé l'école, ainsi que nous allons le rappeler, comme un levier permettant d'assurer les "principes de liberté, d'égalité, d'universalité et de droit à l'instruction» (Charlot, 1987, p. 50). Ceci ne signifie nullement que nous nous considérons qu'il s'agit là de la seule fonction sociale de l'institution scolaire. Voir à cet égard Bourdieu (1967). Maints autres chercheurs ont montré qu'elle assumait diverses fonctions sociales, de socialisation, de sélection, de reproduction, d'homogénéisation, etc.

11 La notion d'individu est inhérente au développement d'une citoyenneté démocratique. Voir sur ce point Gourevitch (1997), Habermas (1991), Potvin, Fournier et Couture (2000).

12 Pour une étude de l'évolution de l'université moderne et de ses différents modèles (scientifique, libéral, pragmatique, de l'excellence), voir entre autres Drèze et Debelle (1968), Lenoir (2000b), Lessard et Tardif (à paraître), Readings (1996).

13 Si le système éducatif français s'appuie sur un processus de sélection progressive des «meilleurs», des plus méritants, «sur la supériorité des esprits» (Goldring, 2000, p. 34), d'où l'importance du concours, le système scolaire aux États-Unis se présente comme non sélectif et discriminatoire, la sélection sociale se jouant cependant lors de l'entrée dans la vie active, dans la compétition (struggle for life), là ou les «meilleurs» sortiront du lot et 
réussiront leur vie. Les deux processus d'inclusion-exclusion sociale recourent ainsi à des modalités opérationnelles distinctes, l'école assumant toutefois une fonction sociale beaucoup plus importante en France. Ce constat est également relevé par Chauvel (2000).

14 Nous ne pouvons débattre ici de cette opposition, déjà traitée à maintes reprises (par exemple, Baker, 1988; Brucy \& Ropé, 2000; Charlot, 1987; Coutel, 1996; Éliard, 1994; Lelièvre, 1994; Nique \& Lelièvre, 1993). Signalons seulement que Sachot (sous presse) note que "pour comprendre la distinction qui est faite dans la langue française entre instruction et éducation, distinction qui va parfois jusqu'à l'opposition, il est nécessaire de se référer aux projets sur l'éducation qui ont été élaborés lors de la Révolution française et, plus particulièrement, au débat qui eut lieu à la suite du Rapport de Condorcet de décembre 1792 au printemps 1793. Cette distinction était ainsi formulée par J.-P. Rabaut Saint-Étienne dans son Projet d'éducation nationale, du 21 décembre 1792: «Il faut distinguer l'instruction publique de l'éducation nationale. L'instruction publique éclaire et exerce l'esprit; l'éducation nationale doit former le cœur: la première doit donner des lumières et la seconde des vertus: la première sera le lustre de la société, la seconde en sera la consistance et la force. L'instruction publique demande des lycées, des collèges, des livres, des instruments de calcul, des méthodes, elle s'enferme dans des murs: l'éducation nationale demande des cirques, des gymnases, des armes, des jeux publics, des fêtes nationales, le concours fraternel de tous les âges et de tous les sexes, et le spectacle imposant et doux de la société humaine rassemblée. Elle veut un grand espace, le spectacle des champs et de la nature; l'éducation nationale est l'aliment nécessaire à tous: l'instruction publique est le partage de quelques-uns. Elles sont sœurs, mais l'éducation nationale est l'aînée. Que dis-je? c'est la mère commune de tous les citoyens, qui leur donne à tous le même lait, qui les élève et les traite en frères, et qui, par la communauté de ses soins, leur donne cet air de ressemblance et de famille qui distingue un peuple ainsi élevé de tous les autres peuples de la terre. Toute la doctrine consiste donc à s'emparer de l'homme dès le berceau, et même avant sa naissance; car l'enfant qui n'est pas né appartient déjà à sa patrie. Elle s'empare de tout l'homme sans le quitter jamais, en sorte que l'éducation nationale n'est pas une institution pour l'enfance, mais pour la vie tout entière. Pour faire bref, [selon cette perspective,] l'éducation inculque, l'instruction est métacritique» (pp. 231-233). Signalons seulement ici la nécessité d'un processus éducatif, au sens entendu par Rabaut Saint-Étienne, puis par Jules Ferry (Lelièvre, 1994; Nique \& Lelièvre, 1993) et Émile Durkheim (1966), en vue de mettre en œuvre un processus de socialisation et d'intégration sociale des futurs citoyens, particulièrement à la fin du $19^{\mathrm{e}}$ siècle où, pour les gens de l'époque, le «salut de la nation républicaine» était en jeu à plus d'un titre. Voir également Chervel (1998).

15 L'érudition était conçue, sous l'Ancien régime, comme «la forme commune du savoir» (Stichweh, 1991, p. 40). Le système des disciplines scientifiques, qui s’installe progressivement à partir du 18e siècle, rendra obsolète l'érudition, perçue dorénavant comme surannée et inadéquate. La formation scolaire revendique dorénavant une formation appuyée sur des savoirs résultant de la recherche disciplinaire.

16 Asher et Malet (1996) considèrent que cette indifférence au regard de la formation pédagogique et professionnelle des enseignants, qui s'appuie sur «leur refus de tenir compte des élèves en tant qu'apprenants, leur concept mythique de professeur charismatique dispensant un savoir sans souci envers ces élèves, bref sur leur réduction de l'acte d'enseignement à une simple transmission de connaissances sans une appréciation indispensable des besoins des élèves, peut être vue comme une forme d'irresponsabilité civique, comme un totalitarisme auto-légitimant qui ne tolère aucune dissension» (p. 277). Voir également Forquin (2001), Kerlan (2001), Prost (1985), Soëtard (2001) et le débat KambouchnerMeirieu (2001). 
17 Nous renvoyons, pour une étude approfondie de la conception de la nation américaine, de ses fondements, de ses discours de légitimation et de son évolution, aux deux excellents livres de Marienstras $(1976,1988)$ et à celui, de facture plus classique sur le plan historique, de Jacquin, Royot et Whitfield (2000).

18 La nation américaine repose sur la conviction qu'elle procède d'un «destin manifeste», celui d'être le «Nouveau peuple d'Israël», avec l'arrivée des Puritains calvinistes dans cette «nouvelle Jérusalem» qu'était l'Amérique du 17 e siècle, d'être les «Pères fondateurs" d'un nouveau "peuple élu» ayant une mission civilisatrice: il s'agit d'œuvrer au bonheur de l'humanité en lui offrant l'exemple américain, en matière de vertu, de liberté et de progrès. Au cœur de ce concept se retrouve la croyance en la supériorité américaine. Cette idée de «destin manifeste" et de supériorité est reprise au $19^{\mathrm{e}}$ siècle pour justifier l'expansionnisme américain à l'intérieur (phase de continentalisation, 1803-1848) et à l'extérieur (phase impérialiste, 1866-1904), l'ethnocide des Amérindiens qui ne sont pas protégés par la Providence (ils meurent des épidémies, les Blancs non) et qui entravent la marche de l'Évangile (c'est-à-dire la conquête de l'Ouest). La justification idéologique (la supériorité anglosaxonne) et théologique (la Providence), renforcée par les mythes de la Conquête de l'Ouest et du melting pot, sont sources de cohésion sociale, mais cette cohésion se définit dans un cadre bien précis (WASP $=$ White-Anglo-Saxon-Protestant) qui procède à l'exclusion des noirs, des Amérindiens, des hispaniques mexicains. Ce ne sont pas des citoyens, mais, au mieux, des résidents (Marienstras, 1988). Le 19e siècle voit l'établissement d'un processus de sécularisation du mythe religieux, mais celui-ci demeure cependant fort vivace, au point où Madeleine Albright pouvait encore déclarer en 1997: «Nous les Américains avons un avantage sur les autres nations. Nous savons qui nous sommes et ce que nous croyons. Nous sommes des constructeurs. Notre responsabilité n'est pas d'agir comme des prisonniers de l'histoire mais de faire l'histoire [...]. Nous avons une raison d'être [..] et la foi que, si nous sommes fidèles à nos principes, nous triompherons». Voir, à nouveau, Marienstras $(1976,1988)$ et aussi, par exemple, le numéro spécial de la revue L'histoire (2000) sur «L'empire américain».

19 Par mentalisme Kliebard (1992a) entend la théorie de la discipline mentale qui postulait que certaines matières (subjects of study) et certaines façons d'étudier ces matières étaient particulièrement utiles pour le développement des capacités mentales (powers of the mind). Par exemple, l'étude des mathématiques était jugée précieuse pour développer les capacités de raisonnement. Plus encore, la théorie du mentalisme prenait position sur la question de différents curriculums pour différentes clientèles scolaires. La question posée était (et elle l'est encore): «Devrait-il y avoir un type de curriculum pour les élèves destinés à l'éducation postsecondaire et un autre pour ceux qui se préparent seulement à la «vie»?» (p. 205). Enfin le mentalisme offrait aussi des indications générales pour l'agencement curriculaire fondé sur une logique naturelle du développement des capacités intellectuelles de l'enfant.

20 Les quatre traditions qui ont marqué au cours du $20^{\mathrm{e}}$ siècle les réformes de la formation à l'enseignement aux États-Unis sont la tradition academique, la tradition axée sur l'efficacité sociale, la tradition développementaliste et la tradition social-reconstructionniste.

21 À ces travaux, il convient d'ajouter de nombreuses recherches, dont, en particulier, celles de Goodlad, Soder \& Sirotnik (1990a, 1990b).

22 Pour Kerr (1995) le «savoir libéral» (en référence à la conception libérale de l'université) est plus que jamais partout en retrait dans les universités américaines qui se soumettent à des stimuli financiers externes.

23 Voir par exemple Barringer (1993), Council of Learned Societies in Education (1996) et National Board for Professional Teaching Standards (1991).

24 Voir par exemple les 19 postulats du renouveau de la formation initiale à l'enseignement retenus par Goodlad, Soder et Sirotnik $(19906)$ et la synthèse de plusieurs propositions chez Lessard, Lenoir, Martin, Tardif et Voyer (1999). 
25 Notons, en passant, que ce constat est tout à fait à l'ordre du jour et se voit encore aujourd'hui validé avec les déclarations du président des États-Unis sur le «monde du mal».

26 L'idée d'excellence serait-elle également arrivée en France, ainsi qu'en témoignerait un livre de l'ex-ministre de l'Éducation nationale, Claude Allègre (1993), L'âge des savoirs: pour une renaissance de l'Université? celui-ci propose entre autres d'ouvrir l'Université aux connaissances afin «de renouveler la recherche et de reconnaître l'excellence» (p. 232).

\section{Références}

Abbott, A. (1991). The order of professionalization: An empirical analysis. Work and Occupations, $18,(4), 355-384$.

Allègre, C. (1993). L'âge des savoirs: pour une renaissance de l'Université. Paris: Gallimard.

Altet, M. (1994). La formation professionnelle des enseignants. Paris: Presses universitaires de France.

Altet, M. (1998). Quelle formation professionnalisante pour développer les compétences de «'enseignement professionnel» et une culture professionnelle d'acteur? In M. Tardif, C. Lessard \& C. Gauthier (Ed.), Formation des maîtres et contextes sociaux. Perspectives internationales (pp. 71-86). Paris: Presses universitaires de France.

Althusser, L. (1976). Idéologie et appareils idéologiques d'État. In L. Althusser, Positions (pp. 67-125). Paris: Éditions sociales (1. éd. 1970).

American Council on Education (1999). Transforming the way teachers are taught. An action agenda for college and university presidents. Washington, DC: ACE.

Anderson, P. (1996). Histoire et leçon du néolibéralisme. La construction d'une voie unique. Page deux, Octobre (téléaccessible à l'adresse URL ‘http://www.fastnet.ch/Page2/p2_neolib_anderson.html).

Ansart, P. (1974). Les idéologies politiques. Paris: Presses universitaires de France.

Aronowitz, S. (2000). Knowledge factory. Dismantling the corporate university and creating true higher learning. Boston, MA: Bacon Press.

Asher, C. \& Malet, R. (1996). The IUFM and initial teacher training in France: Socio-political issues and the cultural divide. Journal of Education for Teaching, 22, (3), 271-281.

Audigier, F. (2001). Quelques questions à l'enseignement de l'histoire aujourd'hui et demain. Le cartable de Clio, I, 55-57.

Bacharan, N. \& Kaspi, A. (2000). L'Amérique est-elle vraiment une démocratie? L’histoire, (248), 73-75.

Baker, M. K. (1988). Condorcet, raison et politique (trad. M. Nobile). Paris: Hermann (1. éd. 1975).

Ball, S. \& Van Zanten, A. (1998). Logiques de marché et éthiques contextualisées dans les systèmes scolaires français et britannique. Éducation et sociétés, 1, 47-71.

Barnabé, C. (1993). La pérestroïka américaine. Revue des sciences de l'éducation, XIX, (3), 569584.

Barringer, M. D. (1993). How the national board builds professionalism. Educational Leadership, 50, (6), 18-22.

Beauchemin, J., Bourque, G., Duchastel, J., Boismenu, G. \& Noël, A. (1995). Présentation. L'État dans la tourmente. Cahiers de la recherche sociologique, 24, 7-13.

Bell, D. (1967). The end of ideology. Glencoe: Free Press.

Bourdieu, P. (1967). Fins et fonctions du système d'enseignement. Les cahiers de l'INAS, 1, 2531.

Bourdoncle, R. (1993). Professionnalisation of teachers and teacher education. Texte d'orientation pour le séminaire de l'ATEE, Barcelone, 1-3 juillet. Bruxelles: Association for Teacher Education in Europe. 
Bourque, G., Duchastel, J. \& Kuzminski, A. (1997). Présentation: Les grandeurs et les misères de la société globale au Québec. Cahiers de la recherche sociologique, 28, 7-17.

Bressoux, P. (2002). Réflexions sur l'effet-maître et l'étude des pratiques enseignantes. Les dossiers des sciences de l'éducation, 5, 35-52.

Bru, M. (1991). Les variations didactiques dans l'organisation des conditions d'apprentissage. Toulouse: Éditions universitaires du Sud.

Bru, M. (1997). Connaître l'acte d'enseigner. Sherbrooke: Université de Sherbrooke, Faculté d'éducation (Documents du LARIDD n ${ }^{\circ} 12$ ).

Brucy, G. \& Ropé, F. (2000). Suffit-il de scolariser? Paris: Éditions de l'Atelier/Éditions ouvrières.

Brune, F. (2000). Sous le soleil de Big Brother. Précis sur «1984» et l'usage des années 2000. Paris: L'Harmattan.

Bulle, N. (1999). La rationalité des décisions scolaires. Analyse comparée de l'évolution de l'enseignement secondaire français et américain au cours $d u X X X^{e}$ siècle. Paris: Presses universitaires de France.

Bulle, N. (2000). L'enseignement secondaire face aux enjeux de la modernisation démocratique. In A. Van Zanten (Ed.), L'école, l'état des savoirs (pp. 365-373). Paris: La Découverte et Syros.

Burbules, N. C. \& Torres, C. A. (Ed.). (2000a). Globalization and education. Critical perspectives. New York, NY: Routledge.

Burbules, N. C. \& Torres, C. A. (2000b). Globalization and education: An introduction. In N. C. Burbules \& C. A. Torres (Ed.), Globalization and education. Critical perspectives (pp. 1-26). New York, NY: Routledge.

Carbonneau, M. (1993). Modèles de formation et professionnalisation de l'enseignement: analyse critique de tendances nord-américaines. Revue des sciences de l'éducation, XIX, (1), 33-57.

Carnegie Task Force on the Education and the Economy Task Force on Teaching as a Profession (1986). A nation prepared: Teachers for the twenty-first century. New York, NY: Carnegie Forum on Education and the Economy.

Carnoy, M. (2000). School choice? Or is it privatization? Educational Researcher, 29, (7), 1520.

Chauvel, L. (2000). Valorisation et dévalorisation sociale des titres scolaires: une comparaison France-États-Unis. In A. Van Zanten (Ed.), L'école, l'état des savoirs (pp. 341-352). Paris: La Découverte et Syros.

Charlot, B. (1987). L'école en mutation. Paris: Payot.

Charlot, B. (Ed.). (1995). Les sciences de l'éducation, un enjeu, un défi. Paris: ESF.

Chartier, A.-N. (1998). L'expertise enseignante entre savoirs pratiques et savoirs théoriques. Recherche et formation, 27, 67-82.

Chervel, A. (1998). La culture scolaire. Une approche historique. Paris: Belin.

Chesnais, F. (1997). La mondialisation du capital. Nouvelle édition augmentée. Paris: Syros.

Clifford, G. J. \& Guthrie, J. W. (1988). Ed school: A brief for professional education. Chicago, IL: The University of Chicago Press.

Condorcet (M. J. A. Caritat, marquis de) (1989a). Écrits sur l'instruction publique - Vol. I: Cinq mémoires sur l'instruction publique (Texte présenté, annoté et commenté par Charles Coutel \& Catherine Kintzler). Paris: Édilig (1. éd. 1791-1792).

Condorcet (M. J. A. Caritat, marquis de) (1989b). Écrits sur l'instruction publique - Vol. II: Rapport sur l'instruction (Texte présenté, annoté et commenté par C. Coutel \& C. Kintzler). Paris: Édilig (1. éd. 1791-1792).

Condorcet (M. J. A. Caritat, marquis de) (1994). Rapport et projet de décret sur l'organisation générale de l'instruction publique présentés à l'Assemblée nationale, au nom du Comité d'instruction publique les 20 et 21 avril 1792. In J. Dumazedier \& É. Donfu, La le- 
çon de Condorcet. Une conception oubliée de l'instruction pour tous nécessaire à une république (pp. 131-173). Paris: L'Harmattan.

Coulby, D. (2000). Beyond the national curriculum. Curricular centralism and cultural diversity in Europe and the USA. London: Routledge.

Council of Learned Societies in Education (1996). Standards for academic and professional instruction in foundations of education, educational studies, and educational policy studies $(2 \mathrm{e}$ éd.). Ann Arbor, MI: CLSE.

Coutel, C. (1996). À l'école de Condorcet. Contre l'orléanisme des esprits. Paris: Ellipse/Éditions Marketing.

Crampe-Casnabet, M. (1985). Condorcet, lecteur des lumières. Paris: Presses universitaires de France.

de Queiroz, J. M. (2000). Pédagogie et pédagogues contre le savoir? In A. Van Zanten (Ed.), L'école, l'état des savoirs (pp. 374-380). Paris: La Découverte et Syros.

de Tocqueville, A. (1968). De la démocratie en Amérique (édité par J.-P. Mayer). Paris: Gallimard (1. éd. 1840).

Dostaler, G. (2001). Le libéralisme de Hayek. Paris: La Découverte.

Doyle, W. (1990). Classroom knowledge as a foundation for teaching. Teachers College Record, 91, (3), 347-360.

Drèze, J. \& Debelle, J. (1968). Conceptions de l'Université. Paris: Éditions universitaires.

Dubet, F. \& Martuccelli, D. (1996). À l'école. Sociologie de l'expérience scolaire. Paris: Seuil.

Durand, M.-J. (1994). État de la question conduisant à la conception d'un réseau notionnel du domaine curriculaire. Thèse de doctorat, Faculté d'éducation, Université du Québec à Montréal.

Durkheim, E. (1966). Éducation et sociologie. Paris: Presses universitaires de France (1. éd. 1922).

Education Commission of the States (1983). Action for excellence: A comprehensive plan to improve our nation's schools. Denver, CO: Education Commission of the States, Task Force on Education for Economic Growth.

Éliard, M. (1994). Modernité de l'idéal démocratique et de la science morale et politique de Condorcet - Exposé. In J. Dumazedier \& É. Donfu (Ed.), La leçon de Condorcet. Une conception oubliée de l'instruction pour tous nécessaire à une république (pp. 64-74). Paris: L'Harmattan.

Fialaire, J. (1996). L'école en Europe. Paris: La Documentation française.

Fichte, J. G. (1967). Plan déductif d'un établissement d'enseignement supérieur à fonder à Berlin. In L. Ferry, J.-P. Person \& A. Renaut (Ed.), Philosophies de l'Université: l'idéalisme allemand et la question de l'Université (pp. 165-252). Paris: Payot (1. éd. 1817).

Forquin, J.-C. (1989). École et culture. Le point de vue des sociologues britanniques. Bruxelles: Éditions universitaires/De Boeck.

Forquin, J.-C. (2001). La pédagogie, la culture et la raison: variations sur un thème d'Ernest Gellner. Revue française de pédagogie, 135, 131-144.

Fournier, R. (1997). Société globale ou société virtuelle: un secret mal gardé. Cahiers de la recherche sociologique, 28, 37-62.

Freitag, M. (1995). Le naufrage de l'Université et autres essais d'épistémologie politique. Montréal et Paris: Nuit blanche/La Découverte.

Freitag, M. (1996). La «technocratisation» de la recherche comme obstacle à l'innovation et aux nouveaux chercheurs. In Y. Lenoir \& M. Laforest (Ed.), La bureaucratisation de la recherche en éducation et en sciences sociales. Constats, impacts et conséquences (pp. 41-62). Sherbrooke: Éditions du CRP.

Freitag, M. \& Pineault, É. (dir). (1999). Le monde enchaîné. Perspectives sur l'AMI et le capitalisme globalisé. Montréal: Éditions Nota Bene.

Gautherin, J. (2000). L'universalisme de l'école laïque à l'épreuve. In A. Van Zanten (Ed.), L'école, l'état des savoirs (pp. 381-388). Paris: La Découverte/Syros. 
Gélinas, J. B. (2000). La globalisation du monde. Laisser faire ou faire? Montréal: Éditions Écosociété.

Geoffroy, Y. \& Lenoir, Y. (2000). Differents rationales at work: Conceptions of the curriculum conception in francophone Europe, in USA, in the German and Scandinavian world and in Quebec. Différentes logiques à l'cuvre: la conception du curriculum dans le monde francophone européen, aux États-Unis, dans le monde germano-scandinave et au Québec. Texte de la communication à la Canadian Association for Curriculum Studies (CACS) / Association canadienne pour l'étude du curriculum (ACEC), $28^{\mathrm{e}}$ Congrès annuel de la Canadian Society for the Study of Education (CSSE) / Société canadienne pour l'étude de l'éducation (SCEE), Edmonton, 24-27 mai.

Geoffroy, Y., Lenoir, Y. \& Hasni, A. (1999). Differing conceptions of interdisciplinarity in education: The american, french and Quebec perspectives. Texte de la communication lors du 21th Annual Association for Integrative Studies Conference, Napierville, Illinois, 30 Septembre-3 octobre.

Goldring, M. (2000). Voie royale, voie républicaine. La formation des élites en France et en Grande-Bretagne. Paris: Syllepse.

Goodlad, J. I., Soder, R. \& Sirotnik, K. A. (1990a). Places where teachers are taught. San Francisco, CA: Jossey-Bass.

Goodlad, J. I., Soder, R. \& Sirotnik, K. A. (1990b). Teachers for our nation's schools. San Francisco, CA: Jossey-Bass.

Gourevitch, A. J. (1997). La naissance de l'individu dans l'Europe médiévale. Paris: Seuil.

Gouvernement de la France (1995). Programmes de l'école primaire. Paris: Ministère de l'Éducation nationale, Direction des écoles, Centre national de documentation pédagogique.

Gouvernement de la France (1989). Loi 89-486 d'orientation sur l'éducation. Bulletin officiel du ministère de l'Éducation nationale, $n^{\circ}$ spécial 4 (31 août 1989), 14.

Green, A. (1990). Education and state formation: The rise of educational systems in England, France and the United States. London: The Macmillan Press.

Gudmundsdottir, S. (1988). Knowledge use among experienced teachers: Four case studies of high school teaching. Thèse de doctorat non publiée, Stanford University, Stanford, CA.

Guiomar, J.-Y. (1990). La nation entre l'histoire et la raison. Paris: La Découverte.

Gundem, B. B. \& Hopmann, S. (Ed.). (1998). Didaktik and/or curriculum. An international dialogue. New York, NY: Peter Lang.

Habermas, J. (1991). La société des individus (trad. de Die Gesellschaft der Individuen). Paris: Fayard.

Hameyer, U., Frey, K., Haft, H. \& Kuebart, F. (Ed.). (1986). Curriculum research in Europe. Berwyn \& Lisse: Swets North America Inc./Swets \& Zeitlinger B. V.

Hobsbawn, E. J. (1992). Nations and nationalism since 1780: Programme, myth, reality (2e éd.). Cambridge: Cambridge University Press.

Holmes Group (1986). Tomorrow's teachers: A report of the Holmes Group. East Lansing, MI: The Holmes Group.

Holmes Group (1990). Tomorrow's schools. Principles for the design of professional development schools. East Lansing, MI: The Holmes Group.

Holmes Group (1995). Tomorrow's schools of education. East Lansing, MI: The Holmes Group.

Hopmann, S. \& Gundem, B. B. (1998). Didaktik meets curriculum: Towards a new agenda. In B. B. Gundem \& S. Hopmann (Ed.), Didaktik andlor curriculum. An international dialogue (pp. 331-354). New York, NY: Peter Lang.

Hopmann, S. \& Riquarts, K. (Ed.). (1995). Starting a dialogue: Roots and issues in the beginning conversation between the didaktik and curriculum traditions. Journal of Curriculum Studies, 27, 3-12.

Horkheimer, M. (1974). Éclipse de la raison suivi de La raison et conservation de soi (trad. J. Debouzy \& J. Laizé). Paris: Payot (1. éd. 1947). 
Horkheimer, M. \& Adorno, T. (1974). La dialectique de la raison. Fragments philosophiques (trad. É Kaufholz). Paris: Payot (1. éd. 1944).

Jacquin, P., Royot, D. \& Whitfield, S. (2000). Le peuple américain. Origines, immigration, ethnicité et identité. Paris: Seuil.

Jackson, P. W. (1992). Conceptions of curriculum and curriculum specialists. In P. W. Jackson (Ed.), Handbook of research on curriculum (pp. 3-40). New York, NY: Macmillan.

Judge, H. (1998). Préface. In M. Fullan, G. Galluzzo, P. Morris \& N. Watson, The rise and stall of teacher education reform (pp. 3-8). Washington, DC: American Association of Colleges for Teacher Education.

Judy, R. (1993). (Dis)forming the american canon: African-arabic slave narratives and the vernacular. Minneapolis, MN: University of Minnesota Press.

Kambouchner, D. \& Meirieu, P. (2001). Débat: Kambouchner / Meirieu. Revue française de pédagogie, 137, 5-16.

Kerlan, A. (2001). À quoi pensent les pédagogues? La pensée pédagogique au miroir du philosophe. Revue française de pédagogie, 137, 17-26.

Kerr, C. (1995). The uses of the university. Cambridge, MA: Harvard University Press.

Kherroubi, M. \& Plaisance, É. (2000). Les évolutions de l'enseignement du premier degré en France. In A. Van Zanten (Ed.), L'école, l'état des savoirs (pp. 26-34). Paris: La Découverte et Syros.

Kintzler, C. (1989). Fonder l'école républicaine dans une cité laïque. In Condorcet (M. J. A. Caritat, marquis de), Écrits sur l'instruction publique - Vol. I: Cinq mémoires sur l'instruction publique (1. éd. 1791-1792) (Texte présenté, annoté et commenté par Charles Coutel et Catherine Kintzler, pp. 9-20). Paris: Édilig.

Klein, J.-L., Tremblay, P.-A. \& Dionne, H. (Ed.). (1997). Au-delà du néolibéralisme. Quel rôle pour les mouvemetns sociaux? Sainte-Foy: Presses de l'Université du Québec.

Kliebard, H. (1986). The struggle for the american curriculum: 1893-1958. Boston, MA: Routledge and Kegan Paul.

Kliebard, H. M. (1992a). Forging the american curriculum. Essays in curriculum history and theory. New York, NY/London: Routledge.

Kliebard, H. M. (1992b). Constructing a history of the american curriculum. In P. H. Jackson (Ed.), Handbook of research on curriculum. A project of the American Educational Research Association (pp. 157-184). New York, NY: Macmillan.

Labaree, D. F. (1992). Power, knowledge, and the rationalization of teaching: A genealogy of the movement to professionalize teaching. Harvard Educational Review, 62, (2), 123-154.

Lacotte, J. \& Lenoir, Y. (1999). Didactics and professional practice in preservice teacher education: A comparison of the situations in France and Quebec. Instructional Science. An International Journal of Learning and Cognition, 27, (1), 165-192.

Lang, V. (1999). La professionnalisation des enseignants. Paris: Presses universitaires de France.

Lang, V. (2000). Les IUFM et la professionnalisation des enseignants. In A. van Zanten (Ed.), L'école, l'état des savoirs (pp. 228-235). Paris: La Découverte.

Lang, V. (2001). La profession enseignante en France: permanence et éclatement. Éducation et francophonie, XXIX (1) (téléaccessible à l'adresse URL http://www.acelf.ca/revue/XXIX$\mathrm{I} /$ /).

Langouët, G. (1994). Modernité de l'idéal démocratique et de la science morale et politique de Condorcet - Introduction. In J. Dumazedier \& É. Donfu (Ed.), La leçon de Condorcet. Une conception oubliée de l'instruction pour tous nécessaire à une république (pp. 61-63). Paris: L'Harmattan.

Lelièvre, C. (1990). Histoire des institutions scolaires, 1789-1989. Paris: Nathan.

Lelièvre, C. (1994). De Condorcet à Ferry et de Ferry à Condorcet - Exposé. In J. Dumazedier \& É. Donfu (Ed.), La leçon de Condorcet. Une conception oubliée de l'instruction pour tous nécessaire à une république (pp. 49-60). Paris: L'Harmattan. 
Lenoir, Y. (1999). Interdisciplinarité. In J. Houssaye (Ed.), Questions pédagogiques. Encyclopédie historique (pp. 291-314). Paris: Hachette.

Lenoir, Y. (2000a). L'Université du $21^{\mathrm{e}}$ siècle: culturelle ou «excellente»? Éducation Canada, 40, (3), 32-36, 47.

Lenoir, Y. (2000b). La recherche en éducation: quelques enjeux à l'aube du $21^{e}$ siècle/Educational research: Some issues at the dawn of the 21st century. Conférence d'ouverture, $13^{\mathrm{e}}$ congrès international de l'Association mondiale des sciences de l'éducation (AMSE) / World Association for Educational Research (WAER), Sherbrooke, 26 juin (téléaccessible à l'adresse URL ‘http://www.educ.usherb.ca/crie//).

Lenoir, Y. (2001a). Logiques européenne francophone et nord-américaine et conceptualisation de l'éducation: de la différenciation dans le cadre des États-nations à une tendance à la dissolution dans le contexte de la mondialisation. Notes de cours. Bruxelles: Université libre de Bruxelles, Faculté des sciences psychologiques et de l'éducation.

Lenoir, Y. (à paraître). Pour une éthique de l'évaluation des résultats en éducation: quelles compatibilités entre les attentes sociales et les visées éducatives? In P. Meirieu \& C. Lessard (Ed.), L'obligation des résultats en éducation. Paris: L'Harmattan.

Lenoir, Y. (sous presse). La question éthique en supervision de stages en enseignement: quelle éthique et pour qui? In M. Boutet \& N. Rousseau (Ed.), Les enjeux de la supervision universitaire des stages en enseignement: aspects éthiques et modes d'évaluation. Québec: Presses de l'Université du Québec.

Léon, A. (1971). De la Révolution française aux débuts de la IIIe République. In M. Debesse \& G. Mialaret (Ed.), Traité des sciences pédagogiques. 2- Histoire de la pédagogie (pp. 331378). Paris: Presses universitaires de France.

Lessard, C., Lenoir, Y., Martin, D., Tardif, M. \& Voyer, B. (1999). La formation des enseignantes et des enseignants: aspects comparatifs et prospectifs. Étude réalisée pour l'ADEREQ. Montréal: CRIFPE.

Lessard, C. \& Tardif, M. (à paraître). La nature et la place d'une formation professionnelle selon les conceptions de l'Université. In Y. Lenoir \& M.-H. Bouillier-Oudot (Ed.), Savoirs professionnels et curriculum de formation de professionnels. Une variété de situations, une varitété de conceptions, une variété de propositions. Sherbrooke et Paris: Éditions du CRP I L'Harmattan.

L'histoire (2000). L'empire américain, 7, 7-106 (Numéro hors série).

Liston, D. P. \& Zeichner, K. M. (1991). Teacher Education and the social conditions of schooling. New York, NY: Routledge.

Luke, A. \& Luke, C. (2000). A situated perspective on cultural globalization. In N. C. Burbules \& C. A. Torres (Ed.), Globalization and education. Critical perspective (pp. 275-297). New York, NY: Routledge.

Marienstras, É. (1976). Les mythes fondateurs de la nation américaine. Paris: François Maspéro.

Marienstras, É. (1988). Nous le peuple. Les origines du nationalisme américain. Paris: Gallimard.

Martinelli, A. (1979). L'impact politique et social des firmes transnationales. Sociologie et sociétés, $X I$, (2), 11-37.

Matsuura, K. (2000). La protection de la diversité culturelle dans une économie en voie de mondialisation. Texte de la conférence du directeur général de l'Unesco lors de la $30^{e}$ Conférence générale de l'Association interparlementaire Canada-France, Assemblée nationale, 12 septembre.

Mercure, D. (Ed.). (2001). Une société-monde? Les dynamiques sociales de la mondialisation. Québec et Bruxelles: Presses de l'Université laval/De Boeck Université.

National Board for Professional Teaching Standards (1991). Toward high and rigorous standards: Initial policies of the National Board for Professional Teaching Standards (3e éd.). Washington, DC: NBPTS. 
National Commission on Excellence in Education (1983). A nation at risk: The imperative for educational reform. Washington, DC: US Government Printing Office.

National Commission on Teaching and America's Future (1996). What matters most: Teaching for America's future. New York, NY: NCTAF.

Newman, J. H. (1925). The idea of university: Defined and illustrated. London: Longmans, Green and Co (1. éd. 1852).

Nique, C. \& Lelièvre, C. (1993). La République n’éduquera plus. Paris: Plon.

Novoa, A. (2001). Commentaires sur la place de la recherche pédagogique. Revue française de pédagogie, 135, 101-105.

Organisation de coopération et de développement économiques (1992). L'OCDE et les indicateurs internationaux de l'enseignement. Un cadre d'analyse. Paris: OCDE.

Organisation de coopération et de développement économiques (1995). Le dernier cycle de l'enseignement obligatoire: Quelle attente? Paris: OCDE.

Organisation for economic co-operation and development/Organisation de coopération et de développement économiques (1997). Prepared for life? How to measure cros-curricular competencies. Prêts pour l'avenir? Comment mesurer les compétences transdisciplinaires. Paris: OECD/OCDE.

Organisation for economic co-operation and development \& Unesco. (2001). Teachers for tomorrow's schools. Analysis of the world education indicators. Paris: Unesco-UIS/OECD.

Parker, F. (1993). Reforming US teacher education in the 1990s. Western Carolina University, School of Education and Psychology (ERIC 58084).

Pasche, C. \& Peters, S. (1997). Les premiers pas de la Société du Mont-Pèlerin ou les dessous chics du néolibéralisme. In D. Le Dinh (Ed.), L'avènement des sciences sociales comme disciplines académiques, $X I X^{e}-X X^{e}$ siècles. Lausanne: Antipodes (coll. «Les annuelles», $\mathrm{n}^{\circ} 8$ ).

Pena-Ruiz, H. (2001). Un totalitarisme tranquille. La démocratie confisquée. Paris: Syllepse.

Petrella, R. (1995). Les nouvelles Tables de la Loi. Le Monde diplomatique, Octobre, 28.

Petrella, R. (2000). L'éducation, victime de cinq pièges. À propos de la société de la connaissance. Montréal: Éditions Fides.

Pettit, P. (1997). Republicanism. A theory of freedom and government. Oxford: Clarendon Press.

Pinar, W. F. (1998). Dreamt into existence by others: Notes on school reform in the US. In M. Tardif, C. Lessard \& C. Gauthier (Ed.), Formation des maîtres et contextes sociaux. Perspectives internationales (pp. 201-229). Paris: Presses universitaires de France.

Popkewitz, T. S. (2000). Reform as the social administration of the child: Globalization of knowledge and power. In N. C. Burbules \& C. A. Torres (Ed.), Globalization and education. Critical perspectives (pp. 157-186). New York, NY: Routledge.

Potvin, M., Fournier, B. \& Couture, Y. (Ed.). (2000). L'individu et le citoyen dans la société moderne. Montréal: Presses de l'Université de Montréal.

Prost, A. (1968). Histoire de l'enseignement en France, 1800-1967. Paris: Armand Colin.

Prost, A. (1985). Éloge des pédagogues. Paris: Seuil.

Prost, A. (1997). Éducation, société et politiques. Une histoire de l'enseignement de 1945 à nos jours. Paris: Seuil (1. éd. 1992).

Rabaut Saint-Étienne, J.-P. (1792). Projet d'éducation nationale. In M. J. A. Caritat, marquis de Condorcet (1989), Écrits sur l'instruction publique. Second volume: Rapport sur l'instruction (pp. 231-235) (Texte présenté, annoté et commenté par Charles Coutel). Paris: Édilig (1. éd. 1792).

Raymond, D. \& Lenoir, Y. (1998). Enseignants de métier et formation initiale: une problématique divergente et complexe. In D. Raymond \& Y. Lenoir (Ed.), Enseignants de métier et formation initiale. Des changements dans les rapports de formation à l'enseignement (pp. 47102). Bruxelles: De Bøck Université.

Readings, B. (1996). The University in ruins. Cambridge, MA: Havard University Press.

Reed, P. L. (1993). An analysis of the professionalization of teaching in the United States. Texte 
de la communication lors du séminaire de l'Association for Teacher Education in Europe (ATEE), Barcelone, 1-3 juillet.

Reid, W. A. (1998). Systems and structures or myths an fables? A cross-cultural perspective on curriculum studies. In B. B. Gundem \& S. Hopmann (Ed.), Didaktik and/or curriculum. An international dialogue (pp. 11-27). New York, NY: Peter Lang.

Robinson, I. (1995). Democratic critiques of the institutions and processes of neoliberal international economic integration: An assessment. Cahiers de la recherche sociologique, 24, 161-183.

Rudolph, F. (1977). Curriculum. A history of the american undergraduate course of study since 1636. San Francisco, CA: Jossey-Bass.

Sachot, M. (2000). La formation professionnelle à l'enseignement entre deux vecteurs intégrateurs: les disciplines et le curriculum. Éducation et francophonie, XXVIII, (2) (téléaccessible à l'adresse URL http://www.acelf.ca/revue/)).

Sachot, M. (sous presse). Disciplines du maître - disciplines de l'élève: contre une "disciplinarisation» du primaire. In M. Sachot \& Y. Lenoir (Ed.), Didactique et interdisciplinarité. La formation didactique des enseignants du primaire: approches disciplinaires ou interdisciplinaires? Québec: Presses de l'Université Laval.

Saltman, K. J. (2000). Collateral damage: Corporatizing public schools. A threat to democracy. Lanham, MD: Rowman \& Littlefield.

Schelling, F. W. J. (1967). Leçons sur la méthode des études académiques. In L. Ferry, J.-P. Person \& A. Renaut (Ed.), Philosophies de l'Université: l'idéalisme allemand et la question de l'Université (pp. 41-164). Paris: Payot (1. éd. 1803).

Schiller, F. (1967). Letters on the aesthetic education of man (trad. E. M. Wilkinson \& L. A. Willoughby). Oxford: Clarendon Press (1. éd. 1793-1794).

Schlegel, F. (1841). Lectures on the history of literature, ancient and modern. New York, NY: Smith and Wright.

Schleiermacher, F. (1979). Pensées de circonstance sur les universités de conception allemande. In L. Ferry, J.-P. Person \& A. Renaut (Ed.), Philosophies de l'Université: l'idéalisme allemand et la question de l'Université (pp. 253-318). Paris: Payot (1. éd. 1808).

Schnapper, D. (1994). La communauté des citoyens. Sur l'idée moderne de nation. Paris: Gallimard.

Schnapper, D. (1999). Traditions nationales et connaissance rationnelle. Sociologie et sociétés, XXXI, (2), 15-26.

Schnapper, D. \& Bachelier, C. (2000). Qu'est-ce que la citoyenneté? Paris: Gallimard.

Schulze, H. (1996). État et nation dans l'histoire de l'Europe. Paris: Seuil.

Shulman, L. S. (1987). Knowledge and teaching. Foundations of the new reform. Harvard Educational Review, 57, (1), 1-22.

Snedden, D. (1921). Sociological determination of objectives in education. Philadelphia, PA: J. B. Lippincott Company.

Soëtard, M. (2001). Qu'est-ce que la pédagogie? Paris: ESF.

Stichweh, R. (1991). Études sur la genèse du système scientifique moderne (Trad. F. Blaise). Lille: Presses universitaires de Lille.

Tanguy, L. (1994). Compétences et intégration sociale dans l'entreprise. In F. Ropé \& L. Tanguy (Ed.), Savoirs et compétences. De l'usage de ces notions dans l'école et l'entreprise (pp. 205235). Paris: L'Harmattan.

Tanner, D. \& Tanner, L. (1990). History of the school curriculum. New York-Londres: Macmillan/Collier Macmillan.

Tardif, M., Lessard, C. \& Gauthier, C. (1998). Formation des maîtres et contextes sociaux. Perspectives internationales. Paris: Presses universitaires de France.

Terrot, N. (1997). Histoire de l'éducation des adultes en France. La part de l'éducation des adultes dans la formation des travailleurs: 1789-1971. Paris: L'Harmattan. 
Thériault, J.-Y. (1997). La société globale est morte... vive la société globale! Cahiers de la recherche sociologique, 28, 19-35.

Thériault, J.-Y. (Ed.). (1999). Citoyenneté et identité sociale. Sociologie et sociétés, XXXI, (2), 5-190 (numéro thématique).

Thiesse, A.-M. (1999). La création des identités nationales. Europe XVIIIe-XXe siècle. Paris: Seuil.

Tillema, H. H. (1995). Changing the professional knowledge and beliefs of teachers: A training study. Learning and Instruction, 5, 291-318.

Tochon, F. V. (Ed.). (1999). Didactics in the french speaking world. Instructional Science. An International Journal of Learning and Cognition, 27, (1), 1-192 (Numéro thématique).

Tremblay, A. (1997). Feu la société globale et les méthodes quantitatives: de nouveaux termes pour un ancien débat? Cahiers de la recherche sociologique, 28, 63-88.

Trottier, C. (1987). La «nouvelle» sociologie de l'éducation en grande-Bretagne: un mouvement de pensée en voie de dissolution? Revue française de pédagogie, 78, 5-20.

Van Haecht, A. (1990). L'école à l'épreuve de la sociologie. Questions à la sociologie de l'éducation. Bruxelles: De Boeck Université.

Vaniscotte, F. (1996). Les écoles de l'Europe. Systèmes éducatifs et dimension européenne. Toulouse et Paris: IUFM de Toulouse/Institut national de recherche pédagogique.

Vigarello, S. (1994). De Condorcet à Ferry et de Ferry à Condorcet - Introduction. In J. Dumazedier et É. Donfu (Ed.), La leçon de Condorcet. Une conception oubliée de l'instruction pour tous nécessaire à une république (pp. 45-48). Paris: L'Harmattan.

von Humboldt, W. (1979). Sur l'organisation interne et externe des établissements scientifiques supérieurs à Berlin. In L. Ferry, J.-P. Person \& A. Renaut (Ed.), Philosophies de l'Université: l'idéalisme allemand et la question de l'Université (pp. 319-329). Paris: Payot (1. éd. 1809).

Vosniadou, S., Ioannides, C., Dimitragopoulou, A. \& Papademetriou, E. (2001). Designing learning environments to promote conceptual change in science. Learning and Instruction, 11, (4-5), 381-419.

Walzer, M. (1997). Sphères de justice. Une défense du pluralisme et de l'égalité. Paris: Seuil.

Warnier, J.-P. (1999). La mondialisation de la culture. Paris: La Découverte.

Whitehead, A. N. (1929). The aims of education. London: Williams and Norgate.

Whitty, G. \& Edwards, T. (1998). School choice policies in England and the United States: An exploration of their origins and significance. Comparative Education, 34, (2), 211-227.

Williams, R. (1965). The long revolution. Harmondsworth: Penguin Books (1. éd. 1961).

Williams, R. (1968). Culture and society, 1780-1950. Harmondsworth: Penguin Books (1. éd. 1958).

Wilson, S. M. \& Berne, J. (1999). Teacher learning and the acquisition of professional knowledge: An examination of research on contemporary professional development. In A. IranNejad \& P. David Pearson (Ed.), Review of research in education (pp. 173-209). Washington, DC: American Educational Research Association.

Zay, D. (1994). La formation des einseignants au partenariat. Une réponse à la demande sociale? Paris: Presses universitaires de France. 


\section{Die aktuellen Reformen der Lehrerbildung in Frankreich und in den USA: Elemente einer sozialhistorischen Kontextuali- sierung auf dem Hintergrund der jeweiligen Erziehungs- konzepte}

\section{Zusammenfassung}

Der Artikel hat das Ziel, die je dominierenden Konzeptionen in der Art und Weise der Aktualisierung der Lehrerbildungssysteme in Frankreich und den USA in einer sozialhistorischen Perspektive aufzuzeigen. Die jeweiligen Dominanzen ergeben sich aus unterschiedlichen Traditionen in der, Konstruktion ' des Erziehungssystems. Wenn diese zwei Traditionen auch ein gemeinsames Ziel haben, nämlich die Emanzipation des Menschen, ein Ziel, das direkt mit der Funktionsweise des Schulsystems in einem modernen "Nationalstaat» zusammenhängt, so ergeben sie sich doch aus einer je unterschiedlichen sozialhistorischen Dynamik und sie aktualisieren sich auf unterschiedliche Art und Weise in ihrer jeweiligen Gesellschaft. Im Kontext des derzeitigen internationalen Austausches, der unter anderem durch die vorherrschende neoliberale Ideologie gefördert wird, die ihrerseits durch verschiedene Organisationen getragen und durch die amerikanische Vormachtstellung gestützt wird, werden Elemente aus der nordamerikanischen Tradition in die Erziehungssysteme der französischsprachigen Länder eingeführt, welche deren herrschende Logik stören und Brüche in deren interner Kohärenz provozieren.

\section{Le attuali riforme della formazione degli insegnanti in Francia e negli Stati Uniti: elementi per una lettura storico-sociale a partire dal concetto di educazione}

\section{Riassunto}

Partendo da una prospettiva storico-sociale, l'articolo mira a fare emergere l'esistenza di elementi dominanti nella concezione e nelle modalità di attualizzazione dei sistemi di formazione degli insegnanti in Francia e negli Stati Uniti. Tali elementi derivano da due tradizioni di implementazione del sistema educativo. Se da un lato queste due tradizioni hanno nell'emancipazione degli esseri umani un obiettivo comune, dall'altro lato risultano da dinamiche storico-sociali distinte e si concretizzano diversamente nelle rispettive realtà sociali. Nel contesto degli scambi internazionali attuali, caratterizzati tra l'altro dall'egemonia del discorso ideologico neoliberale e dalla pressione americana, l'inserimento di elementi derivanti dalla tradizione statunitense nel sistema educativo francese comportano per quest'ultimo effetti di disturbo e di rottura della coerenza interna. 


\section{Current reforms in teacher education occurring in France and the United States: placing socio-historical elements in perspective, starting with the concept of education}

\section{Summary}

Supported by a socio-historical perspective, the purpose of this article is to highlight the existing leading characteristics in the conception of actualized modalities of the teacher education systems in France and the United States. These dominant conceptions emerge from two traditions initiated in their respective educational systems. Both traditions have a common foundation in the emancipation of human beings, directly linked to the function of a modern nation-state school system and created from distinct socio-historical dynamics, and are actualized differently in their respective society. In the context of current international exchange, characterized by, among other things, a neoliberal hegemonistic ideology, an approach used by a diversity of organizations and supported by the North American influence, the introduction of these elements of the North American argument into the French educational system could be the source of important disequilibrium, thus disrupting its current perspective and provoking ruptures in the current internal coherence of the European French speaking educational system. 